\title{
INFINITE-DIMENSIONAL DIFFUSIONS AS LIMITS OF RANDOM WALKS ON PARTITIONS
}

\author{
Alexei Borodin and Grigori Olshanski
}

\begin{abstract}
Starting with finite Markov chains on partitions of a natural number $n$ we construct, via a scaling limit transition as $n \rightarrow \infty$, a family of infinite-dimensional diffusion processes. The limit processes are ergodic; their stationary distributions, the so-called $\mathrm{z}$-measures, appeared earlier in the problem of harmonic analysis for the infinite symmetric group. The generators of the processes are explicitly described.

Keywords: Diffusion processes; Thoma's simplex; Infinite symmetric group; Schur functions; $\mathrm{z}-$-measures; Dirichlet forms
\end{abstract}

Mathematics Subject Classification (2000): 60J60; 60C05

\section{CONTEnTs}

Introduction

1. The abstract formalism

2. A toy example: the Pascal triangle

3. The $\mathrm{z}-$ measures

4. The "up" and "down" operators for the z-measures

5. Convergence of Markov semigroups

6. The pre-generator $A_{z, z^{\prime}}$ as a differential operator

7. The limit process

References

\section{INTRODUCTION}

The present paper originated from our previous study of the problem of harmonic analysis on the infinite symmetric group $S_{\infty}$. This problem leads to a family $\left\{P_{z}\right\}$ of probability measures, the $\mathrm{z}$-measures, which depend on the complex parameter $z \in \mathbb{C}$. The $\mathrm{z}-$ measures live on the Thoma simplex, an infinite-dimensional compact space $\Omega$ which is a kind of dual object to the group $S_{\infty}$. The aim of the paper is to introduce stochastic dynamics related to the z-measures. Namely, we construct a family $\left\{\boldsymbol{\omega}_{z}(t)\right\}$ of diffusion processes in $\Omega$ indexed by the same parameter $z \in \mathbb{C}$. The processes $\left\{\boldsymbol{\omega}_{z}(t)\right\}$ are obtained from certain Markov chains on partitions of $n$ in a scaling limit as $n \rightarrow \infty$. These Markov chains arise in a natural way, due to the approximation of the group $S_{\infty}$ by the increasing chain of the finite symmetric groups $S_{n}$. Each z-measure $P_{z}$ serves as a unique invariant distribution for the corresponding process $\boldsymbol{\omega}_{z}(t)$, and the process $\boldsymbol{\omega}_{z}(t)$ is ergodic with respect to $P_{z}$. Moreover, $P_{z}$ is a symmetrizing measure, so that $\boldsymbol{\omega}_{z}(t)$ is reversible. We describe 
the spectrum of the generator of the process $\boldsymbol{\omega}_{z}(t)$ and compute the associated (pre)Dirichlet form.

As was shown in our previous papers, the $\mathrm{z}$-measures and associated random point processes (that is, measures on infinite point configurations) are similar to random point processes coming from random matrix ensembles. A remarkable fact is that this similarity extends to related dynamical (that is, time-dependent) models. In particular, the dynamical model that we study in the present paper is similar to time-dependent random point processes (like the Dyson Brownian motion) coming from random matrices.

Now let us describe our results and their origin in more detail.

The Thoma simplex. This is the subspace $\Omega \subset[0,1]^{\infty} \times[0,1]^{\infty}$ consisting of couples $(\alpha, \beta)$, where $\alpha$ and $\beta$ are two infinite sequences of nonincreasing nonnegative real numbers, $\alpha_{1} \geq \alpha_{2} \geq \cdots \geq 0$ and $\beta_{1} \geq \beta_{2} \geq \cdots \geq 0$, such that $\sum \alpha_{i}+\sum \beta_{i} \leq 1$. Define the infinite symmetric group $S_{\infty}$ as the union of the chain $S_{1} \subset S_{2} \subset S_{3} \subset \ldots$ of finite symmetric groups. By Thoma's theorem [T], 1964, the points $\omega=(\alpha, \beta) \in \Omega$ parameterize the indecomposable normalized characters of the group $S_{\infty}$ (in a hidden form, this remarkable result is contained in an earlier paper by Edrei [Ed], 1952). In this sense $\Omega$ may be regarded as a dual object to $S_{\infty}$

Origin of the $\mathbf{z}-$ measures. The problem of harmonic analysis for the group $S_{\infty}$, as stated by Kerov, Olshanski, and Vershik [KOV1], consists in decomposing the socalled generalized regular representations $\mathbf{T}_{z}$. These representations depend on an arbitrary complex number $z$ and form a deformation of the biregular representation of the group $S_{\infty} \times S_{\infty}$ in $\ell^{2}\left(S_{\infty}\right)$. As shown in [KOV2], the decomposition of $\mathbf{T}_{z}$ into irreducibles is uniquely determined by an equivalence class $\mathbf{P}_{z}$ of Borel measures on $\Omega$; moreover, except the coincidence $\mathbf{P}_{z}=\mathbf{P}_{\bar{z}}$, these classes are mutually singular. ${ }^{1}$ The structure of $\mathbf{P}_{z}$ substantially depends on whether $z$ is an integral point or not. The former case was studied in [KOV2]; it turns out that for each $z \in \mathbb{Z}, \mathbf{P}_{z}$ is supported by a countable union of finite-dimensional faces of the simplex $\Omega$. The latter case, $z \in \mathbb{C} \backslash \mathbb{Z}$, which is of primary interest for us, is more complex. However, in this case, the construction of $\mathbf{T}_{z}$ provides a distinguished measure in the class $\mathbf{P}_{z}$, which is our z-measure $P_{z}{ }^{2}$ The $\mathrm{z}$-measures were studied in detail in a series of our papers, see [BO1-7].

Measures on Young diagrams. Let $\mathbb{Y}_{n}$ denote the set of Young diagrams with $n$ boxes. Recall that there is a bijective correspondence between the Young diagrams $\lambda \in \mathbb{Y}_{n}$ and the irreducible characters of $S_{n}$, so that $\mathbb{Y}_{n}$ may be regarded as the dual object to $S_{n}$. The construction of the present paper relies on the fact that any probability measure $P$ on $\Omega$ comes with a canonical approximation by a sequence $\left\{M_{n}\right\}$ of probability measures carried by finite sets $\mathbb{Y}_{n}$. Note that as $n$ gets large, the finite sets $\mathbb{Y}_{n}$ approximate, in an appropriate way, the space $\Omega$. This intuitively agrees with the fact that the infinite group $S_{\infty}$ is approximated by the finite groups $S_{n}$. Furthermore, for each $n$, the embedding $S_{n} \hookrightarrow S_{n+1}$ induces a canonical Markov transition kernel $p_{n+1, n}^{\downarrow}$ from $\mathbb{Y}_{n+1}$ to $\mathbb{Y}_{n}$. It turns out that $M_{n}$ and $M_{n+1}$

\footnotetext{
${ }^{1}$ The latter fact is not too surprising because the carrying space $\Omega$ is infinite dimensional.

${ }^{2}$ Actually, the definition of the $\mathrm{z}$-measures can be extended to a larger set of values of the parameter, see $\S 3$ below. According to this, starting from $\S 3$ we use, instead of the single subscript $z$, two subscripts $z, z^{\prime}$.
} 
are always consistent with this kernel: its application to $M_{n+1}$ gives $M_{n}$. This can be written as $M_{n+1} p_{n+1, n}^{\downarrow}=M_{n}$; here and below we let a kernel act on a measure on the right. There exists another transition kernel, denoted as $p_{n, n+1}^{\uparrow}$, such that, conversely, $M_{n} p_{n, n+1}^{\uparrow}=M_{n+1}$.

This picture can also be described as follows: Any sequence $\left\{M_{n}\right\}$ of probability measures consistent with the "down" transition kernels can be viewed as the law of a Markov sequence $\left\{\lambda^{(n)}\right\}$ of random variables such that, for each $n=1,2, \ldots$, the $n$th variable $\lambda^{(n)}$ takes values in $\mathbb{Y}_{n}$ and is distributed according to $M_{n}$, while the "down" transition kernel $p_{n+1, n}^{\downarrow}$ describes the conditional distribution of $\lambda^{(n)}$ given $\lambda^{(n+1)}$. Then the "up" transition kernel $p_{n, n+1}^{\uparrow}$ determines the conditional distribution of $\lambda^{(n+1)}$ given $\lambda^{(n)}{ }^{3}$

It should be noted that although the "down" and "up" transition kernels play symmetric roles, there is a substantial difference between them, for the "down" kernel is a canonical object associated with the inductive limit group $S_{\infty}=\varliminf_{\text {, }} S_{n}$, while the "up" kernel varies depending on the concrete sequence $\left\{M_{n}\right\}$.

The "up-down" Markov chains. The starting point of our construction is the observation that for any $n$ one can build a "natural" Markov chain in $\mathbb{Y}_{n}$ with stationary distribution $M_{n}$. We call it the $n$th up-down chain: By definition, its transition operator $T_{n}$ is defined as the superposition $T_{n}=p_{n, n+1}^{\uparrow} \circ p_{n+1, n}^{\downarrow}$ (the order of reading is from left to right when $T_{n}$ is applied to measures, and from right to left when applied to functions). Note that if $(\ldots, \lambda(t-1), \lambda(t), \lambda(t+1), \ldots)$ stands for a trajectory of our Markov chain in $\mathbb{Y}_{n}$ then for any time moment $t$, the Young diagrams $\lambda(t)$ and $\lambda(t+1)$ either coincide or differ from each other by a minimal possible transformation preserving the total number of boxes: displacement of a single boundary box to a new position.

One can introduce the graph with the vertex set $\mathbb{Y}_{n}$ and the edges formed by couples of Young diagrams such that their symmetric difference consists of two boxes. Our chain jumps along the edges and so it is a random walk on this graph.

Now the idea is to look at the limit behavior of the up-down Markov chains as $n \rightarrow \infty$. We do not know what can be said in the abstract context, i.e., when $\left\{M_{n}\right\}$ comes from an arbitrary probability measure $P$ on $\Omega$, but in the concrete case of the z-measures $P=P_{z}$ we are able to prove the convergence of the chains to continuous time Markov processes in $\Omega$ which turn out to be diffusion processes. Here the limit transition assumes appropriate scalings both of space (from $\mathbb{Y}_{n}$ to $\Omega$ ) and of time (from discrete to continuous). The space scaling is the same as that leading from $M_{n}$ to $P$ : roughly speaking, it consists in shrinking a diagram $\lambda \in \mathbb{Y}_{n}$ by the factor of $n^{-1}$. The time scaling makes one step of the $n$th chain with large number $n$ equivalent to a small time increment $\Delta t \approx n^{-2}$.

Note that, instead of the up-down chains, we could equally well deal with the down-up chains corresponding to the transition operators of the form $p_{n, n-1}^{\downarrow}$ 。 $p_{n-1, n}^{\uparrow}$. At the finite level one gets slightly different chains but the limit process does not change.

\footnotetext{
${ }^{3}$ In this context, the Thoma simplex appears as the entrance boundary for the sequence $\left\{\lambda^{(n)}\right\}$ viewed as a Markov chain with discrete time $n$ ranging in reverse direction, from $+\infty$ to 1 , see [KOO]. This Markov chain is a little bit unusual and highly non-homogeneous: its state space varies with time. Nevertheless, the theory of boundaries (see, e.g., [KSK]) can be readily adapted to such a situation.
} 
The above definition of the up-down and down-up Markov chains does not pretend to originality. The second named author learnt it long ago from Sergei Kerov (but Kerov never published it). In a different context, such Markov chains appeared in Fulman's work [F]: he was interested in the Plancherel distributions $M_{n}$ which correspond to the case when $P$ is the delta measure at the distinguished point $(\alpha \equiv 0, \beta \equiv 0)$ of $\Omega$. Quite possibly, the trick is well known, for it is very simple and can be applied to any Markov sequence $\left(\ldots, \xi^{(n-1)}, \xi^{(n)}, \xi^{(n+1)}, \ldots\right)$ of random variables with values in possibly varying spaces.

Limit transition. Our analysis of the large $n$ asymptotics of the up-down Markov chains uses the technique explained in Ethier-Kurtz [EK2] and goes as follows. Let $C(\Omega)$ be the Banach space of continuous functions with the supremum norm on the compact space $\Omega$. We embed $\mathbb{Y}_{n}$ into $\Omega$ by means of a map first introduced by Vershik and Kerov, which gives us a projection of $C(\Omega)$ onto the (finite-dimensional) Banach space $C\left(\mathbb{Y}_{n}\right)$. The Markov operator $T_{n}$ can be viewed as an operator in the latter space. We show that, in an appropriate sense,

$$
\lim _{n \rightarrow \infty} n^{2}\left(T_{n}-1\right)=A,
$$

where $A$ is a closable, dissipative operator $A$ in $C(\Omega)$ with dense range. By the Hille-Yosida theorem and a general theorem due to Kurtz, the closure $\bar{A}$ of the operator $A$ generates a contractive semigroup $\{T(t)\}$ in $C(\Omega)$, and moreover, we have convergence of semigroups:

$$
\lim _{n \rightarrow \infty} T_{n}^{\left[n^{2} t\right]}=T(t) .
$$

This implies that the limit semigroup $\{T(t)\}$ is positivity preserving. Consequently, it determines a Feller Markov process in $\Omega$, which is our process $\boldsymbol{\omega}_{z}(t)$.

The key moment of this argument is the existence of the limit operator $A$ with desired properties. Surprisingly enough, we can prove this by purely algebraic tools, without any analytical machinery. Specifically, we are dealing with a nice algebra of functions on the set $\mathbb{Y}:=\cup \mathbb{Y}_{n}$ of all Young diagrams. This algebra, introduced in $[\mathrm{KO}]$, is isomorphic to the algebra $\Lambda$ of symmetric functions, and it turns out that all necessary computations can be carried out by manipulations with symmetric functions. Here we substantially use the so-called Frobenius-Schur symmetric functions introduced in [OlRV] (they are essentially the same as the shifted Schur functions [OO]). The domain of $A$, $\operatorname{Dom}(A) \subset C(\Omega)$, can be identified with a quotient $\Lambda^{\circ}$ of the algebra $\Lambda$ by an ideal; this quotient can be embedded into $C(\Omega)$ as a dense subspace. It turns out that both $A$ and all $T_{n}$ 's can be viewed as operators in $\Lambda^{\circ} \subset C(\Omega)$ preserving a natural ascending filtration of $\Lambda^{\circ}$ by finite-dimensional subspaces. Due to this fact, the limit transition (0.1) can be understood at the level of linear algebra: as convergence of operators in these finite-dimensional subspaces.

Results about the limit processes. We prove that $\boldsymbol{\omega}_{z}(t)$ is a diffusion process, that is, a strong Markov process with continuous sample paths.

We show that $\boldsymbol{\omega}_{z}(t)$ has the z-measure $P_{z}$ as a unique stationary distribution. Moreover $P_{z}$ is also a symmetrizing measure, so that the process is reversible.

We describe explicitly the spectrum of the generator, which turns out to be discrete. Due to existence of a spectral gap, the process is ergodic. 
We describe the pre-generator $A=A_{z}$ in two different ways: (1) as an operator in the algebra $\Lambda^{\circ}$ acting on the (images of) the Schur functions and (2) as an infinite-dimensional differential operator in appropriate coordinates, which we call the moment coordinates. It is worth noting that these are not the natural coordinates $\alpha_{i}, \beta_{i}$ in $\Omega$.

We represent the quadratic form associated to $A_{z}$ as an integral against $P_{z}$ of a "carré du champs", which does not depend on the parameter and admits a simple expression in the moment coordinates.

Lifting. Let $\widetilde{\Omega}$ denote the cone over $\Omega$. Each of the processes $\boldsymbol{\omega}_{z}(t)$ admits a "lifting" to the cone $\widetilde{\Omega}$. The result is a Markov process $\widetilde{\boldsymbol{\omega}}_{z}(t)$ in $\widetilde{\Omega}$ which is related to $\boldsymbol{\omega}_{z}(t)$ by a skew product construction, like the Brownian motions in the Euclidean space and in its unit sphere. It turns out that, as an equilibrium process, $\widetilde{\boldsymbol{\omega}}_{z}(t)$ is a time dependent determinantal process: its dynamical (i.e., space-time) correlation functions are principal minors of a kernel. That kernel appeared in our paper [BO6] as the result of a scaling limit transition from some continuous time jump Markov processes on Young diagrams. The paper [BO6] left open the question whether the Markov property persists in that limit transition; now we can settle this question affirmatively. We aim to discuss this issue in a subsequent paper.

Diffusions in Kingman's simplex. Diffusion processes in simplices (both in finite and infinite dimensions) were extensively discussed in the literature in connection to mathematical models of population genetics. The paper by Ethier and Kurtz [EK1] is of special interest to us. These authors studied diffusions on the so-called Kingman simplex, which can be identified with the subspace of points in $\Omega$ with all beta-coordinates equal to 0 . On Kingman's simplex, there exists a remarkable family of probability measures, the Poisson-Dirichlet distributions; the Ethier-Kurtz diffusions preserve these distributions. As shown in Petrov $[\mathrm{P}]$, the Ethier-Kurtz diffusions can be constructed by the limit transition from appropriate up-down Markov chains; moreover, the same approach can be extended to a larger family of diffusions on Kingman's simplex, which are associated to Pitman's generalization of the Poisson-Dirichlet distributions. Note that the original construction of [EK1] was quite different. As will be shown in a subsequent paper, both the Ethier-Kurtz diffusions and our processes $\boldsymbol{\omega}_{z}(t)$ are particular cases of a more general construction. This explains the origin of a striking similarity between the both families. We would like to gratefully acknowledge the influence of the paper [EK1] (and of the subsequent paper by Schmuland $[\mathrm{S}]$ ) on our work.

Organization of the paper. In $\S 1$ we present the construction of Markov processes from up-down Markov chains in an abstract form. Our aim here is to single out the formal part of the argument, which can be applied in other situations, outside the context of the present paper. In $\S 2$ we illustrate the abstract formalism on a toy example: here the limit Markov process is a diffusion on $[0,1]$. In $\S 3$ we introduce the necessary material related to the z-measures. In $\S 4$ we compute the action of the transition operators $p_{n+1, n}^{\downarrow}$ and $p_{n, n+1}^{\uparrow}$ on symmetric functions realized as functions on Young diagrams. Using this computation, we show in $\S 5$ that the assumptions of $\S 1$ are verified for the Markov chain related to the $\mathrm{z}$-measures; this proves the existence of the limit processes $\boldsymbol{\omega}_{z}(t)$. In $\S 6$ we derive an alternative expression for the pre-generator, which is used in the next section. In $\S 7$, we prove that $\boldsymbol{\omega}_{z}(t)$ has continuous sample paths and establish other properties of this 
process.

Acknowledgement. The present research was supported by the CRDF grant RUM1-2622-ST-04 (both authors), by the NSF grants DMS-0402047 and DMS0707163 (A. Borodin), and by the RFBR grant 07-01-91209 and SFB 701, University of Bielefeld (G. Olshanski). G. Olshanski is deeply grateful to Yuri Kondratiev and Michael Röckner for hospitality in Bielefeld and fruitful discussions.

\section{The ABSTRACT FORMALism}

Let $L$ be a graded set, that is, $L$ is the disjoint union of subsets $L_{n}$, where $n=0,1,2, \ldots$. Elements of $L$ will be denoted by the letters $\lambda, \mu, \nu$. If $\lambda \in L_{n}$ then we set $|\lambda|=n$. We assume that $L_{0}$ is a singleton and all $L_{n}$ are finite. ${ }^{4}$ We also assume that we are given a function $p^{\downarrow}(\lambda, \mu)$ on $L \times L$ such that:

- $p^{\downarrow}(\lambda, \mu) \geq 0$ for all $\lambda, \mu$;

- $p^{\downarrow}(\lambda, \mu)$ vanishes unless $|\lambda|=|\mu|+1$;

- for every fixed $\lambda$ with $|\lambda| \geq 1$, we have

$$
\sum_{\mu \in L_{|\lambda|-1}} p^{\downarrow}(\lambda, \mu)=1
$$

Thus, the restriction of $p^{\downarrow}(\lambda, \mu)$ to $L_{n} \times L_{n-1}$ is a stochastic matrix for every $n \geq 1$. We may view this matrix as a transition function from $L_{n}$ to $L_{n-1}$. For this reason we call $p^{\downarrow}$ the "down" transition function.

A sequence $M=\left\{M_{0}, M_{1}, \ldots\right\}$, where $M_{n}$ is a probability measure on $L_{n}$, will be called a coherent system (of measures) if for any $n \geq 1$, the measures $M_{n}$ and $M_{n-1}$ are consistent with the transition function from $L_{n}$ to $L_{n-1}$ :

$$
\sum_{\lambda \in L_{n}} M_{n}(\lambda) p^{\downarrow}(\lambda, \mu)=M_{n-1}(\mu) \quad \text { for any } \mu \in L_{n-1},
$$

where $M_{n}(\lambda)$ means the measure of the singleton $\{\lambda\}$.

Let $M=\left\{M_{n}\right\}$ be a coherent system such that supp $M_{n}$, the support of $M_{n}$, is the whole $L_{n}$ for each $n$, that is, $M_{n}(\lambda)>0$ for all $n$ and all $\lambda \in L_{n}$. Then we define the "up" transition function $p^{\uparrow}$ as follows:

$$
p^{\uparrow}(\lambda, \nu)=\frac{M_{n+1}(\nu)}{M_{n}(\lambda)} p^{\downarrow}(\nu, \lambda), \quad n=|\lambda|, \quad \nu \in L_{n+1} .
$$

Note that $M=\left\{M_{n}\right\}$ determines a family of random variables indexed by $n=$ $0,1, \ldots$ such that the $n$th variable $\lambda^{(n)}$ takes the values in $L_{n}$ and has the law $M_{n}$, and, moreover,

$$
\operatorname{Prob}\left\{\lambda^{(n-1)}=\mu \mid \lambda^{(n)}=\lambda\right\}=p^{\downarrow}(\lambda, \mu)
$$

for each $n \geq 1$. In these terms, the "up" transition function is interpreted as the conditional probability

$$
p^{\uparrow}(\lambda, \nu)=\operatorname{Prob}\left\{\lambda^{(n+1)}=\nu \mid \lambda^{(n)}=\lambda\right\} .
$$

This implies that $p^{\uparrow}$ is a stochastic matrix of format $L_{n} \times L_{n+1}$ for any $n=0,1, \ldots$

Note also that each couple $\left(M_{n}, M_{n+1}\right)$ is consistent with the "up" transition function:

$$
\sum_{\lambda \in L_{n}} M_{n}(\lambda) p^{\uparrow}(\lambda, \nu)=M_{n+1}(\nu) \quad \text { for any } \nu \in L_{n+1} .
$$

\footnotetext{
${ }^{4}$ The latter assumption could be relaxed but it is sufficient for the purpose of the present work.
} 
Definition 1.1. Let $M=\left\{M_{n}\right\}$ be a coherent system with $\operatorname{supp} M_{n}=L_{n}$. For any $n$ we define a Markov operator $T_{n}$ on the set $L_{n}$ as the composition of the "up" and "down" transition functions, from $L_{n}$ to $L_{n+1}$ and then back to $L_{n}$. The matrix of $T_{n}$ is given by:

$$
T_{n}(\lambda, \tilde{\lambda})=\sum_{\nu \in L_{n+1}} p^{\uparrow}(\lambda, \nu) p^{\downarrow}(\nu, \tilde{\lambda}), \quad \lambda, \tilde{\lambda} \in L_{n} .
$$

The operator $T_{n}$ defines a Markov chain on the set $L_{n}$. We call this Markov chain the ( $n$th level) up-down chain. ${ }^{5}$

Proposition 1.2. The measure $M_{n}$ is an invariant distribution for the nth level up-down Markov chain. Moreover, the chain is reversible with respect to $M_{n}$.

Proof. The first claim is evident from (1.1) and (1.3). Indeed, $p_{n, n+1}^{\uparrow}$ sends $M_{n}$ to $M_{n+1}$ and then $p_{n+1, n}^{\downarrow}$ returns $M_{n+1}$ back to $M_{n}$. To prove the second claim we have to check that the $L_{n} \times L_{n}$ matrix $M_{n}(\lambda) T_{n}(\lambda, \tilde{\lambda})$ is symmetric. From the definition of $T_{n}$ and using (1.2) we get

$$
M_{n}(\lambda) T_{n}(\lambda, \widetilde{\lambda})=\sum_{\nu \in L_{n+1}} M_{n+1}(\nu) p_{n+1, n}^{\downarrow}(\nu, \lambda) p_{n+1, n}^{\downarrow}(\nu, \widetilde{\lambda}),
$$

which is symmetric.

We aim to study the behavior of the up-down chains as $n \rightarrow \infty$. To do this we choose a topological space $\bar{L}$ and embeddings $\iota_{n}: L_{n} \hookrightarrow \bar{L}$, and we make an appropriate limit transition inside $\bar{L}$. As a result we obtain a continuous time Markov process in $\bar{L}$.

An excellent reference about limit transitions from Markov chains to continuous time Markov processes is Ethier-Kurtz's book [EK2]. We use some general facts from [EK2] but we also explore some specific properties of our model. ${ }^{6}$ Namely, in our concrete situation the following assumptions hold true:

(A1) The ambient space $\bar{L}$ is a compact, metrizable, separable topological space. Below we denote by $C(\bar{L})$ the Banach space of continuous real functions with the canonical norm

$$
\|f\|=\sup _{\omega \in \bar{L}}|f(\omega)| .
$$

(A2) The sets $\iota_{n}\left(L_{n}\right)$ approximate $\bar{L}$ in the following sense: any open subset of $\bar{L}$ has a nonempty intersection with $\iota_{n}\left(L_{n}\right)$ for all $n$ large enough.

(A3) There is a distinguished dense subspace $\mathcal{F}$ of the Banach space $C(\bar{L})$ and an ascending exhaustive filtration $\left(\mathcal{F}^{m}\right)$ of $\mathcal{F}$ by finite-dimensional subspaces such that each $\mathcal{F}^{m}$ is invariant under the Markov operators $T_{n}$ in the following sense:

Denote by $C\left(L_{n}\right)$ the (finite-dimensional) Banach space of functions on $L_{n}$ with the norm

$$
\|g\|_{n}=\sup _{\lambda \in L_{n}}|g(\lambda)|
$$

\footnotetext{
${ }^{5}$ Likewise, interchanging the transition functions, one could introduce the down-up chains. In the concrete situation studied in the present paper, the down-up chains slightly differ from the up-down ones but have the same limit.

${ }^{6}$ The motivation for the concrete choice of the data $\left(L=\left(L_{n}\right), p^{\downarrow}, M=\left\{M_{n}\right\}, \bar{L}, \iota_{n}\right)$ comes from Vershik-Kerov's theory [VK], [K].
} 
and let $\pi_{n}: C(\bar{L}) \rightarrow C\left(L_{n}\right)$ be defined by

$$
\left(\pi_{n}(f)\right)(\lambda)=f\left(\iota_{n}(\lambda)\right), \quad \lambda \in L_{n}, \quad f \in C(\bar{L}) .
$$

Observe that $\pi_{n}$ is injective on $\mathcal{F}^{m}$ provided that $n$ is large enough, where the necessary lower bound on $n$ depends on $m .^{7}$ Then the invariance property means that, for any $m, \pi_{n}\left(\mathcal{F}^{m}\right)$ is invariant under $T_{n}$, at least for large enough $n$.

Thus, identifying $\mathcal{F}^{m}$ and $\pi_{n}\left(\mathcal{F}^{m}\right)$, which makes sense for large $n$, one may say that the operators $T_{n}$ leave the spaces $\mathcal{F}^{m}$ invariant.

(A4) There is a sequence $\left\{\varepsilon_{n}\right\}$ of positive numbers converging to 0 such that, under the identification $\mathcal{F}^{m}=\pi_{n}\left(\mathcal{F}^{m}\right)$, the limit

$$
\lim _{n \rightarrow \infty} \varepsilon_{n}^{-1}\left(T_{n}-\mathbf{1}\right) f=A f .
$$

exists in any finite-dimensional space $\mathcal{F}^{m}$. Here 1 stands for the identity operator. Clearly, we get in this way a limit operator $A: \mathcal{F} \rightarrow \mathcal{F}$ which preserves each subspace $\mathcal{F}^{m}$.

(A5) $\mathcal{F}$ contains the constant function 1 .

Recall that a conservative Markov semigroup in $C(\bar{L})$ is a strongly continuous semigroup $\{T(t)\}_{t \geq 0}$ of contractive operators in $C(\bar{L})$ preserving the cone of nonnegative functions and the constant function 1 .

Definition 1.3. Following [EK2, Ch. 1, Section 6], let us say that a sequence of functions $\left\{f_{n} \in C\left(L_{n}\right)\right\}$ converges to a function $f \in C(\bar{L})$ if $\left\|f_{n}-\pi_{n}(f)\right\|_{n} \rightarrow 0$. Then we write $f_{n} \rightarrow f$.

Observe that $\left\|\pi_{n}(f)\right\|_{n} \leq\|f\|$ and, by virtue of $(\mathrm{A} 2),\left\|\pi_{n}(f)\right\|_{n} \rightarrow\|f\|$. Again by (A2), a sequence $\left\{f_{n} \in C\left(L_{n}\right)\right\}$ may have at most one limit in $C(\bar{L})$.

Proposition 1.4. Assume that the assumptions (A1)-(A5) stated above are satisfied.

(1) The operator $A: \mathcal{F} \rightarrow \mathcal{F}$ defined in (A4) is closable in the Banach space $C(\bar{L})$.

(2) The closure $\bar{A}$ of $A$ generates a conservative Markov semigroup $\{T(t)\}$ in $C(\bar{L})$.

(3) The discrete semigroup $\left\{1, T_{n}, T_{n}^{2}, T_{n}^{3}, \ldots\right\}$ converges, as $n \rightarrow \infty$, to $\{T(t)\}$ in the following sense:

$$
T_{n}^{\left[\varepsilon_{n}^{-1} t\right]} \pi_{n}(f) \rightarrow T(t) f, \quad \forall f \in C(\bar{L}),
$$

for all $t \geq 0$, uniformly on bounded intervals, where the limit is understood according to Definition 1.3.

We will call $A$ the pre-generator of the semigroup $\{T(t)\}$.

Proof. Step 1. The operator $A: \mathcal{F} \rightarrow \mathcal{F}$ is dissipative, that is, $\|(s \mathbf{1}-A) f\| \geq s\|f\|$ for any $s \geq 0$ and $f \in \mathcal{F}$.

Indeed, fix $m$ so large that $f \in \mathcal{F}^{m}$. Assuming $n$ large enough, we may identify $\mathcal{F}^{m}$ with its image under $\pi_{n}$ and view $T_{n}$ as an operator in $\mathcal{F}_{m}$. Set $A_{n}=\varepsilon_{n}^{-1}\left(T_{n}-\right.$

\footnotetext{
${ }^{7}$ This is true because of (A2) and the fact that $\operatorname{dim} \mathcal{F}^{m}<\infty$.
} 
1). Since $T_{n}$ is a contraction with respect to the norm $\|\cdot\|_{n}$, the operator $T_{n}-\mathbf{1}$ is dissipative with respect to this norm, whence the same holds for $A_{n}$. Since $A_{n} \rightarrow A$ in the finite-dimensional space $\mathcal{F}^{m}$ and since $\|g\|_{n} \rightarrow\|g\|$ for any $g \in \mathcal{F}^{m}$ we conclude that $A$ is dissipative.

Step 2. By virtue of step 1 , for any $s>0$ and any $m$, the operator $s \mathbf{1}-A$ maps the finite-dimensional subspace $\mathcal{F}^{m}$ onto itself. Thus, $(s \mathbf{1}-A) \mathcal{F}=\mathcal{F}$.

Step 3. Since $A$ is dissipative (step 1 ) and its domain $\mathcal{F}$ is dense, $A$ is closable in $C(\bar{L})$; moreover, for any $s>0$, the closure of the range of $s \mathbf{1}-A$ coincides with the range of $s \mathbf{1}-\bar{A}$, where $\bar{A}$ denotes the closure of $A$ ([EK2, Ch. 1, Lemma 2.11]). Applying step 2 we see that the range of $s \mathbf{1}-\bar{A}$ coincides with the whole space $C(\bar{L})$. Thus, the operator $\bar{A}$ satisfies the assumptions of the Hille-Yosida theorem and therefore it generates a strongly continuous contractive semigroup $\{T(t)\}$ in $C(\bar{L})$, see [EK2, Ch. 1, Thm. 2.6].

Step 4. It follows from (A5) that the constant function 1 is in the domain of $\bar{A}$ and $\bar{A} 1=0$, whence $T(t) 1=1$.

Step 5. All the assumptions of [EK2, Ch. 1, Thm. 6.5] are satisfied. Namely:

- $\{T(t)\}$ is a strongly continuous semigroup of contractions with generator $\bar{A}$;

- the subspace $\mathcal{F}$ is an essential domain for $\bar{A}$, that is, the operator $\bar{A}$ coincides with the closure of its restriction to $\mathcal{F}$;

- each $T_{n}$ is a contraction, and we have $\varepsilon_{n}^{-1}\left(T_{n}-\mathbf{1}\right) \pi_{n}(f) \rightarrow \bar{A} f$ for any $f \in \mathcal{F}$ in the sense of Definition 1.2.

Applying this theorem from [EK2], we obtain (1.4).

Step 6. Finally, (1.4) implies that the operators $T(t)$ preserve nonnegative functions, because the $T_{n}$ 's possess this property (here we again use (A2)). Thus, $\{T(t)\}$ is a Markov semigroup.

Proposition 1.5. The semigroup $\{T(t)\}$ constructed in Proposition 1.4 gives rise to a strong Markov process $X(t)$ in $\bar{L}$. The process has càdlàg sample paths and can start from any point or any probability distribution.

Proof. This is a well-known general fact, see e.g. [EK2, Ch. 4, Thm. 2.7].

Proposition 1.6. Assume additionally that the measures $\iota_{n}\left(M_{n}\right)$ weakly converge to a measure $P$ on $\bar{L}$. Then $P$ is an invariant distribution for the process $X(t)$.

Proof. This directly follows from (1.4). Indeed, let $\langle\cdot\rangle_{P}$ or $\langle\cdot\rangle_{M_{n}}$ means expectation with respect to $P$ or $M_{n}$. The invariance property of $P$ means that

$$
\langle T(t) f\rangle_{P}=\langle f\rangle_{P}, \quad \forall f \in C(\bar{L}), \quad \forall t \geq 0 .
$$

Since $\iota_{n}\left(M_{n}\right)$ weakly converges to $P$, this is equivalent to

$$
\left.\lim _{n \rightarrow \infty}\langle T(t) f)\right\rangle_{\iota_{n}\left(M_{n}\right)}=\lim _{n \rightarrow \infty}\langle f\rangle_{\iota_{n}\left(M_{n}\right)},
$$

which can be rewritten as

$$
\lim _{n \rightarrow \infty}\left\langle\pi_{n}(T(t) f)\right\rangle_{M_{n}}=\lim _{n \rightarrow \infty}\left\langle\pi_{n}(f)\right\rangle_{M_{n}} .
$$

By virtue of (1.4), as $n$ gets large, $\pi_{n}(T(t) f)$ is close in norm to $T_{n}^{\left[\varepsilon_{n}^{-1} t\right]} \pi_{n}(f)$, whence the last limit relation is equivalent to

$$
\lim _{n \rightarrow \infty}\left\langle T_{n}^{\left[\varepsilon_{n}^{-1} t\right]} \pi_{n}(f)\right\rangle_{M_{n}}=\lim _{n \rightarrow \infty}\left\langle\pi_{n}(f)\right\rangle_{M_{n}}
$$


which holds for trivial reasons, because

$$
\left\langle T_{n}^{\left[\varepsilon_{n}^{-1} t\right]} \pi_{n}(f)\right\rangle_{M_{n}}=\left\langle\pi_{n}(f)\right\rangle_{M_{n}}
$$

due to invariance of $M_{n}$ with respect to $T_{n}$.

Proposition 1.7. Under the hypothesis of Proposition 1.6, the pre-generator A: $\mathcal{F} \rightarrow \mathcal{F}$ is symmetric with respect to the inner product

$$
(f, g):=\langle f \cdot g\rangle_{P} .
$$

Proof. The argument is similar to that used in Proposition 1.6. Let us show that

$$
\langle A f \cdot g\rangle_{P}=\lim _{n \rightarrow \infty}\left\langle\varepsilon_{n}^{-1}\left(T_{n}-\mathbf{1}\right) \pi_{n}(f) \cdot \pi_{n}(g)\right\rangle_{M_{n}}, \quad f, g \in \mathcal{F} .
$$

By virtue of Proposition 1.2, the right-hand side is symmetric with respect to $f \leftrightarrow g$, hence the above equality implies the desired symmetry of the left-hand side. We have

$$
\begin{gathered}
\langle A f \cdot g\rangle_{P}=\lim _{n \rightarrow \infty}\langle A f \cdot g\rangle_{\iota_{n}\left(M_{n}\right)}=\lim _{n \rightarrow \infty}\left\langle\pi_{n}(A f \cdot g)\right\rangle_{\iota_{n}\left(M_{n}\right)} \\
=\lim _{n \rightarrow \infty}\left\langle\pi_{n}(A f) \cdot \pi_{n}(g)\right\rangle_{\iota_{n}\left(M_{n}\right)}=\lim _{n \rightarrow \infty}\left\langle\varepsilon_{n}^{-1}\left(T_{n}-\mathbf{1}\right) \pi_{n}(f) \cdot \pi_{n}(g)\right\rangle_{M_{n}},
\end{gathered}
$$

where the last step is justified using (A4).

Proposition 1.8. Under the hypothesis of Proposition 1.6, consider $X(t)$ as an equilibrium process with respect to its invariant distribution $P$. Likewise, consider the up-down Markov chains in equilibrium with respect to the invariant distributions $M_{n}$.

Then the finite-dimensional distributions for the nth chain converge, as $n \rightarrow \infty$, to the corresponding finite-dimensional distributions of the process $X(t)$. Here we assume a natural scaling of time: one step of the nth Markov chain corresponds to a small time interval of order $\Delta t=\varepsilon_{n}$.

Proof. The argument is similar to that used in Proposition 1.6.

\section{A toy example: the Pascal triangle}

Here we illustrate the above formalism on a simple example: the Pascal triangle.

In this example, the set $L$ consists of arbitrary couples $\lambda=(a, b)$ of nonnegative integers. The grading is defined as $|(a, b)|=a+b$. Thus, $L_{0}$ consists of the single point $(0,0), L_{1}$ consists of two points $(0,1)$ and $(1,0), \ldots, L_{n}$ consists of $n+1$ points $(0, n), \ldots,(n, 0)$.

The "down" transition function is defined as follows

$$
p^{\downarrow}((a, b),(a-1, b))=\frac{a}{a+b}, \quad p^{\downarrow}((a, b),(a, b-1))=\frac{b}{a+b},
$$

with all other transitions being of probability 0 . 
As $M_{n}$ we take the uniform measure on the set $L_{n}$. The coherency condition (1.1) is immediately checked. Thus, $M=\left\{M_{n}\right\}$ is a coherent system. The nonzero values of the "up" transition function are

$$
p^{\uparrow}((a, b),(a+1, b))=\frac{a+1}{a+b+2}, \quad p^{\uparrow}((a, b),(a, b+1))=\frac{b+1}{a+b+2} .
$$

The Markov operator $T_{n}$ of the up-down Markov chain is given by the following matrix (we list the nonzero entries only and assume $a+b=n$ )

$$
\begin{aligned}
T_{n}((a, b),(a+1, b-1)) & =\frac{(a+1) b}{(a+b+2)(a+b+1)} \\
T_{n}((a, b),(a-1, b+1)) & =\frac{a(b+1)}{(a+b+2)(a+b+1)} \\
T_{n}((a, b),(a, b)) & =\frac{(a+1)^{2}+(b+1)^{2}}{(a+b+2)(a+b+1)} .
\end{aligned}
$$

The ambient compact space is the closed unit interval $[0,1]$. The embeddings $L_{n} \hookrightarrow \bar{L}$ are defined as $(a, b) \mapsto x$ with $x=\frac{a}{a+b} \in[0,1]$.

The dense subspace $\mathcal{F} \subset C(\bar{L})=C([0,1])$ is the space of polynomials with the canonical filtration by degree.

The fulfilment of (A1), (A2), and (A5) is evident, let us verify (A3) and (A4).

The nontrivial one-step transitions of our Markov chain $(a, b) \rightarrow(a+1, b-1)$ and $(a, b) \rightarrow(a-1, b+1)$ turn into $x \rightarrow x \pm \Delta x$ with $\Delta x=n^{-1}$, where we assume $n=a+b$. According to the above formulas for $T_{n}$,

$$
\begin{aligned}
& \operatorname{Prob}\{x \rightarrow x+\Delta x\}=\frac{(a+1) b}{(a+b+2)(a+b+1)} \\
& \operatorname{Prob}\{x \rightarrow x-\Delta x\}=\frac{a(b+1)}{(a+b+2)(a+b+1)},
\end{aligned}
$$

which can be rewritten as

$$
\begin{aligned}
& \operatorname{Prob}\{x \rightarrow x+\Delta x\}=\frac{n^{2}}{(n+1)(n+2)} x(1-x)+\frac{n}{(n+1)(n+2)}(1-x) \\
& \operatorname{Prob}\{x \rightarrow x-\Delta x\}=\frac{n^{2}}{(n+1)(n+2)} x(1-x)+\frac{n}{(n+1)(n+2)} x .
\end{aligned}
$$

It follows that for a polynomial $f(x)$,

$$
\begin{gathered}
\left(\left(T_{n}-\mathbf{1}\right) f\right)(x)=\frac{n^{2}}{(n+1)(n+2)} x(1-x)(f(x+\Delta x)+f(x-\Delta x)-2 f(x)) \\
+\frac{n}{(n+1)(n+2)}(1-x)(f(x+\Delta x)-f(x))+\frac{n}{(n+1)(n+2)} x(f(x-\Delta x)-f(x))
\end{gathered}
$$

(recall that 1 stands for the identity operator). Therefore,

$$
\begin{gathered}
\left(\left(T_{n}-\mathbf{1}\right) f\right)(x)=\frac{n^{2} \cdot(\Delta x)^{2}}{(n+1)(n+2)} x(1-x)\left(f^{\prime \prime}(x)+\ldots\right) \\
+\frac{n \cdot \Delta x}{(n+1)(n+2)}(1-x)\left(f^{\prime}(x)+\ldots\right)+\frac{n \cdot \Delta x}{(n+1)(n+2)} x\left(-f^{\prime}(x)+\ldots\right),
\end{gathered}
$$


where dots mean higher derivatives multiplied by suitable nonzero powers of $\Delta x$.

From this expression we see that the operator $T_{n}$ is well defined on polynomials and it does not raise the degree, so that assumption (A3) holds true.

Next, we see that if $\varepsilon_{n} \sim n^{-2}$ then, as $n \rightarrow \infty$,

$$
\varepsilon_{n}^{-1}\left(\left(T_{n}-\mathbf{1}\right) f\right)(x) \quad \rightarrow \quad x(1-x) f^{\prime \prime}(x)+(1-2 x) f^{\prime}(x) .
$$

Therefore, assumption (A4) holds with

$$
A=x(1-x) \frac{d^{2}}{d x^{2}}+(1-2 x) \frac{d}{d x} .
$$

Thus, all necessary assumptions are satisfied and one can apply Proposition 1.4 to conclude that our Markov chains converge to a continuous time Markov process $X(t)$ on $[0,1]$. The generator $\bar{A}$ of $X(t)$ is the closure of the differential operator $A$ initially defined on polynomials.

\section{THE Z-MEASURES}

Here we specify the abstract data described in $\S 1$ and introduce related extra notation. For more detail, see [Ol1], [KOV2], [KOO], [BO3].

3.1. Young diagrams and modified Frobenius coordinates. As $L$ we take the set $\mathbb{Y}$ of all Young diagrams including the empty diagram $\varnothing$. The subset $L_{n} \subset L$ becomes the subset $\mathbb{Y}_{n} \subset \mathbb{Y}$ of diagrams with $n$ boxes.

Given $\lambda \in \mathbb{Y}_{n}$, denote by $a_{1}, \ldots, a_{d}, b_{1}, \ldots, b_{d}$ its modified Frobenius coordinates: here $d$ is the number of diagonal boxes in $\lambda, a_{i}$ equals $\frac{1}{2}$ plus the number of boxes in the $i$ th row to the right of the diagonal, and $b_{i}$ equals $\frac{1}{2}$ plus the number of boxes in the $i$ th column below the diagonal. Note that $\sum\left(a_{i}+b_{i}\right)=n$. We write $\lambda=\left(a_{1}, \ldots, a_{d} \mid b_{1}, \ldots, b_{d}\right)$.

If $\lambda$ and $\mu$ are two Young diagrams then we write $\mu \nearrow \lambda$ or, equivalently, $\lambda \searrow \mu$ if $\mu \subset \lambda$ and $|\lambda|=|\mu|+1$. That is, $\lambda$ is obtained from $\mu$ by adding a box. This box is then denoted as $\lambda / \mu$. In terms of the modified Frobenius coordinates, $\mu \nearrow \lambda$ means that $\lambda$ is obtained from $\mu$ either by adding 1 to one of the coordinates or by creating a new pair of coordinates $\left(\frac{1}{2} ; \frac{1}{2}\right.$ ) (the latter happens if the new box $\lambda / \mu$ lies on the diagonal).

More generally, for any $\mu \subset \lambda$ we denote by $\lambda / \mu$ the corresponding skew Young diagram.

3.2. The "down" transition functions. The choice of the "down" transition function $p^{\downarrow}$ is motivated by the representation theory of the symmetric groups $S_{n}$. Recall that $\mathbb{Y}_{n}$ is the set of labels of irreducible representations of $S_{n}$. Given $\lambda \in \mathbb{Y}_{n}$, we denote by $\pi_{\lambda}$ the corresponding irreducible representation of $S_{n}$ and we write $\operatorname{dim} \lambda=\operatorname{dim} \pi_{\lambda}$. Here is an explicit expression for this quantity in terms of the modified Frobenius coordinates:

$$
\frac{\operatorname{dim} \lambda}{n !}=\frac{\prod_{1 \leq i<j \leq d}\left(a_{i}-a_{j}\right)\left(b_{i}-b_{j}\right)}{\prod_{1 \leq i, j \leq d}\left(a_{i}+b_{j}\right) \prod_{1 \leq i \leq d}\left(a_{i}-\frac{1}{2}\right) !\left(b_{i}-\frac{1}{2}\right) !} .
$$

We realize $S_{n}$ as the group of permutations of the set $\{1, \ldots, n\}$, and we embed $S_{n-1}$ into $S_{n}$ as the subgroup fixing the point $n$. The Young rule says that the 
restriction of $\pi_{\lambda}$ (where $\lambda \in \mathbb{Y}_{n}$ ) to $S_{n-1} \subset S_{n}$ decomposes into the multiplicity free direct sum of the representations $\pi_{\mu}$ such that $\mu \nearrow \lambda$. Consequently,

$$
\operatorname{dim} \lambda=\sum_{\mu: \mu \nearrow \lambda} \operatorname{dim} \mu, \quad|\lambda| \geq 2 .
$$

We use this identity to define $p^{\downarrow}$ :

$$
p^{\downarrow}(\lambda, \mu)= \begin{cases}\frac{\operatorname{dim} \mu}{\operatorname{dim} \lambda}, & \mu \nearrow \lambda \\ 0, & \text { otherwise }\end{cases}
$$

and we also set $p^{\downarrow}(\lambda, \mu)=1$ when $|\lambda|=1$ and $|\mu|=0$, that is, when $\lambda$ consists of a single box and $\mu$ is empty.

3.3. The Thoma simplex. As $\bar{L}$ we take the Thoma simplex. Recall that this is the subspace $\Omega \subset[0,1]^{\infty} \times[0,1]^{\infty}$ formed by couples $\omega=(\alpha, \beta)$ such that

$$
\begin{gathered}
\alpha=\left(\alpha_{1} \geq \alpha_{2} \geq \cdots \geq 0\right) \in[0,1]^{\infty}, \quad \beta=\left(\beta_{1} \geq \beta_{2} \geq \cdots \geq 0\right) \in[0,1]^{\infty}, \\
\sum_{i=1}^{\infty}\left(\alpha_{i}+\beta_{i}\right) \leq 1 .
\end{gathered}
$$

The embedding $\iota_{n}: \mathbb{Y}_{n} \hookrightarrow \Omega$ is defined as follows. For $\lambda=\left(a_{1}, \ldots, a_{d} \mid\right.$ $\left.b_{1}, \ldots, b_{d}\right) \in \mathbb{Y}_{n}$ (here we wrote $\lambda$ in terms of the modified Frobenius coordinates), its image $\iota_{n}(\lambda)=(\alpha, \beta)$ is given by

$$
\alpha_{i}=\left\{\begin{array}{ll}
a_{i} / n, & 1 \leq i \leq d, \\
0, & i>d ;
\end{array} \quad \beta_{i}= \begin{cases}b_{i} / n, & 1 \leq i \leq d \\
0, & i>d\end{cases}\right.
$$

The embeddings $\iota_{n}$ satisfy the assumption (A2) of $\S 1$.

3.4. Thoma's measures and moment coordinates. To any point $\omega=(\alpha, \beta) \in$ $\Omega$ one can assign a probability measure $\nu_{\omega}$ on the closed interval $[-1,1]$ :

$$
\nu_{\omega}=\sum_{i=1}^{\infty} \alpha_{i} \delta_{\alpha_{i}}+\sum_{i=1}^{\infty} \beta_{i} \delta_{-\beta_{i}}+\gamma \delta_{0}, \quad \gamma:=1-\sum \alpha_{i}-\sum \beta_{i},
$$

where $\delta_{x}$ denotes the Dirac measure at $x$. The measure $\nu_{\omega}$ is called the Thoma measure corresponding to $\omega$.

Denote by $q_{k}=q_{k}(\omega)$ the moments of $\nu_{\omega}$ :

$$
q_{k}:=\int x^{k} \nu_{\omega}(d x)=\sum_{i=1}^{\infty} \alpha_{i}^{k+1}+(-1)^{k} \sum_{i=1}^{\infty} \beta_{i}^{k+1}, \quad k=1,2, \ldots
$$

and note that the 0 th moment is always equal to 1 . We call $q_{1}, q_{2}, \ldots$ the moment coordinates of $\omega$. Observe that they are continuous functions in $\omega$. Indeed, since $\alpha_{i}$ 's decrease, the condition $\sum \alpha_{i} \leq 1$ implies $\alpha_{i} \leq i^{-1}$ for any $i=1,2, \ldots$, 
whence $\alpha_{i}^{k+1} \leq i^{-k-1}$. Similarly, $\beta_{i}^{k+1} \leq i^{-k-1}$. It follows that the both series are uniformly convergent in $\omega \in \Omega$, which implies their continuity as functions on $\Omega .{ }^{8}$

Let $\mathcal{M}_{1}[-1,1]$ denote the space of probability Borel measures on $[-1,1]$ equipped with the weak topology. Since this topology is determined by convergence of moments, the assignment $\omega \mapsto \nu_{\omega}$ determines a homeomorphism of the Thoma simplex on a compact subset of $\mathcal{M}_{1}[-1,1]$.

On the other hand, the assignment $\omega \mapsto\left(q_{1}, q_{2}, \ldots\right)$ determines a homeomorphism of $\Omega$ on a compact subset of $[-1,1]^{\infty}$.

Thinking of $\Omega$ as of a subspace of $\mathcal{M}_{1}[-1,1]$ or $[-1,1]^{\infty}$ turns out to be useful even though we cannot describe the image of $\Omega$ in $[-1,1]^{\infty}$ explicitly.

Note also that the moment coordinates are algebraically independent as functions on $\Omega$. Indeed, this holds even we restrict them on the subset with all $\beta_{i}$ 's equal to 0 . It follows that the algebra of polynomials $\mathbb{R}\left[q_{1}, q_{2}, \ldots\right]$ can be viewed as a subalgebra of $C(\Omega)$, the (real) Banach algebra of continuous functions on $\Omega$ with pointwise operations and the supremum norm. Since this subalgebra separates points, it is dense in $C(\Omega)$.

3.5. Symmetric functions. Let $\Lambda$ be the algebra of symmetric functions [Ma]. Recall that $\Lambda$ is freely generated (as a commutative unital algebra) by the Newton power sums $p_{1}, p_{2}, \ldots$ As the base field, it is convenient for us to take $\mathbb{R}$. A distinguished basis in $\Lambda$ is formed by the Schur functions $s_{\mu}$ (here and below $\mu$ ranges over $\mathbb{Y})$.

Let $\Lambda^{\circ}=\Lambda /\left(p_{1}-1\right)$ be the quotient of the algebra $\Lambda$ by the ideal generated by $p_{1}-1$. The algebra $\Lambda^{\circ}$ has a natural structure of a filtered algebra inherited from $\Lambda$, and the graded algebra associated to $\Lambda^{\circ}$ is isomorphic to $\Lambda /\left(p_{1}\right)$.

Given $f \in \Lambda$ we denote its image in $\Lambda^{\circ}$ by $f^{\circ}$. In particular, we will be dealing with the elements $s_{\mu}^{\circ} \in \Lambda^{\circ}$ coming from the Schur functions and the elements $p_{k}^{\circ} \in \Lambda^{\circ}$ coming from the Newton power sums. Clearly, $p_{1}^{\circ}=1$ and $\Lambda^{\circ}$ is freely generated (as a unital commutative algebra) by $p_{2}^{\circ}, p_{3}^{\circ}, \ldots{ }^{9}$

Setting $p_{2}^{\circ} \rightarrow q_{1}, p_{3}^{\circ} \rightarrow q_{2}, \ldots$, where $q_{k}=q_{k}(\omega)$ are the moment coordinates defined above, we define an algebra isomorphism between $\Lambda^{\circ}$ and the subalgebra $\mathbb{R}\left[q_{1}, q_{2}, \ldots\right] \subset C(\Omega)$. Thus, each element $f^{\circ} \in \Lambda^{\circ}$ becomes a continuous function $f^{\circ}(\omega)$ on $\Omega$, in particular,

$$
p_{k}^{\circ}(\omega)=\sum_{i=1}^{\infty} \alpha_{i}^{k}+(-1)^{k-1} \sum_{i=1}^{\infty} \beta_{i}^{k}, \quad \omega=(\alpha, \beta) \in \Omega, \quad k=2,3, \ldots
$$

Note that $p_{1}^{\circ} \equiv 1$.

We will take $\Lambda^{\circ}$ as the dense subspace in $C(\Omega)$ required in the assumption (A3) of $\S 1$.

3.6. Boundary measures. Let $\left\{M_{n}\right\}$ be an arbitrary coherent system of probability measures on the sets $\mathbb{Y}_{n}$ with respect to the "down" transition functions introduced in $\S 3.2$; see $\S 1$ for the general definition of coherent systems. Denote by $\iota_{n}\left(M_{n}\right)$ the measure on $\Omega$ obtained as the push-forward of $M_{n}$ with respect to the embedding $\iota_{n}: \mathbb{Y}_{n} \rightarrow \Omega$ defined in $\S 3.3$.

\footnotetext{
${ }^{8}$ This argument substantially relies on the fact that $k+1 \geq 2$. Note that the function $\omega \mapsto$ $\sum \alpha_{i}+\sum \beta_{i}$ is not continuous on $\Omega$.

${ }^{9}$ One could identify $\Lambda^{\circ}$ with the subalgebra in $\Lambda$ generated by $p_{2}, p_{3}, \ldots$ but we do not want to do this.
} 
Theorem. There exists a weak limit $P=\lim _{n \rightarrow \infty} \iota_{n}\left(M_{n}\right)$ on $\Omega$. Conversely, $\left\{M_{n}\right\}$ can be reconstructed from $P$ by means of the equation

$$
M_{n}(\nu)=\operatorname{dim} \nu \int_{\Omega} s_{\nu}^{\circ}(\omega) P(d \omega), \quad \nu \in \mathbb{Y}_{n} .
$$

The correspondence $\left\{M_{n}\right\} \rightarrow P$ is a bijection between coherent systems on $\mathbb{Y}=\cup \mathbb{Y}_{n}$ and probability measures on $\Omega$.

Recall that $s_{\nu}^{\circ}$ stands for the image in $\Lambda^{\circ}$ of the Schur function $s_{\nu} \in \Lambda$. Since $\Lambda^{\circ}$ is embedded in $C(\Omega)$, the value $s_{\nu}^{\circ}(\omega)$ at a point $\omega \in \Omega$ is well defined.

We call $P$ the boundary measure of the coherent system $\left\{M_{n}\right\}$.

This fundamental result is a refinement of Thoma's theorem [T]. It is essentially due to Vershik and Kerov [VK], $[\mathrm{K}]$. See $[\mathrm{KOO}]$ for a detailed proof.

3.7. The $\mathbf{z}-$ measures. Now we proceed to the definition of a distinguished family of coherent systems. Introduce the notation

$$
(z)_{\lambda}=\prod_{(i, j) \in \lambda} z+j-i, \quad \lambda \in \mathbb{Y}, \quad z \in \mathbb{C},
$$

where " $(i, j) \in \lambda$ " means that the product is taken over the boxes of $\lambda$; here and below we denote by $(i, j)$ the box with row number $i$ and column number $j$. The difference $j-i$ is called the content of a box $(i, j)$.

We define likewise $(z)_{\lambda / \mu}$ for skew diagrams $\lambda / \mu$ (then the product is taken over the boxes in $\lambda / \mu$ ). This is a generalization of the Pochhammer symbol

$$
(z)_{n}=z(z+1) \ldots(z+n-1)
$$

which is obtained in the particular case when $\lambda$ is $(n)$, the one-row diagram with $n$ boxes.

Let $z$ and $z^{\prime}$ be complex numbers such that $z z^{\prime} \notin\{0,-1,-2, \ldots\}$. The $z$-measure on the finite set $\mathbb{Y}_{n}$ is the complex measure $M_{z, z^{\prime}}^{(n)}$ with the weights

$$
M_{z, z^{\prime}}^{(n)}(\lambda)=\frac{(z)_{\lambda}\left(z^{\prime}\right)_{\lambda}}{\left(z z^{\prime}\right)_{n}} \frac{(\operatorname{dim} \lambda)^{2}}{n !}, \quad \lambda \in \mathbb{Y}_{n}
$$

The z-measure does not change under transposition $z \leftrightarrow z^{\prime}$. Thus, instead of $z$ and $z^{\prime}$, one can also take as parameters $z z^{\prime}$ and $z+z^{\prime}$.

It is known that the weights sum to 1 ,

$$
\sum_{\lambda \in \mathbb{Y}_{n}} M_{z, z^{\prime}}^{(n)}(\lambda)=1
$$

and that the $\mathrm{z}-$ measures satisfy (1.1):

$$
\sum_{\lambda \searrow \mu} M_{z, z^{\prime}}^{(n)}(\lambda) p^{\downarrow}(\lambda, \mu)=M_{z, z^{\prime}}^{(n-1)}(\mu)
$$

This follows from the representation-theoretical construction of [KOV2]. For a direct proof, see, e.g., [Ol1]. 
The weights $M_{z, z^{\prime}}^{(n)}(\lambda)$ are strictly positive for all $n$ if and only if the couple $\left(z, z^{\prime}\right)$ belongs to one of the following two sets in $\mathbb{C}^{2}$ :

- Principal series: Both $z$ and $z^{\prime}$ are not real and are conjugate to each other.

- Complementary series: Both $z$ and $z^{\prime}$ are real and are contained in the same open interval of the form $(N, N+1)$, where $N \in \mathbb{Z}$.

The union of these two sets admits a nice description in terms of the coordinates

$$
x=\frac{z+z^{\prime}}{2}, \quad y=z z^{\prime}-x^{2}=-\left(\frac{z-z^{\prime}}{2}\right)^{2} .
$$

Namely, this is the domain $\mathcal{D}$ in the real $(x, y)$-plane bounded from below by the piecewise smooth curve $C$ built from countably many smooth $\operatorname{arcs} C_{N}$ : here $N$ ranges over $\mathbb{Z}$ and each $C_{N}$ is an arc of a parabola: $y=-(x-N)^{2},|x-N| \leq \frac{1}{2}$. The principal series and the complementary series are described by points $(x, y) \in \mathcal{D}$ with $y>0$ and $y \leq 0$, respectively.

Thus, to each value $\left(z, z^{\prime}\right)$ of the principal/complementary series (equivalently, to each $(x, y) \in \mathcal{D})$, a coherent family $\left\{M_{n}=M_{z, z^{\prime}}^{(n)}\right\}$ of probability measures is attached. Note that the support of $M_{n}$ is the whole set $\mathbb{Y}_{n}$.

The "up" transition function for the $\mathrm{z}$-measures looks as follows: for $\lambda \in \mathbb{Y}_{n}$

$$
p_{z, z^{\prime}}^{\uparrow}\left(\lambda, \lambda^{\bullet}\right)= \begin{cases}\frac{(z)_{\lambda \bullet / \lambda}\left(z^{\prime}\right)_{\lambda} \bullet / \lambda}{z z^{\prime}+n} \frac{\operatorname{dim} \lambda^{\bullet}}{(n+1) \operatorname{dim} \lambda}, & \lambda^{\bullet} \searrow \lambda, \\ 0, & \text { otherwise. }\end{cases}
$$

We will often use the symbol $\lambda^{\bullet}$ to denote a diagram $\nu$ such that $\nu \searrow \lambda$. Likewise

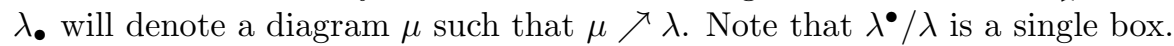

3.8. The boundary $z$-measures. Given a coherent system $\left\{M_{z, z^{\prime}}^{(n)}\right\}$ of $z-$ measures, we denote by $P_{z, z^{\prime}}$ the corresponding boundary measure on $\Omega$ and call it the boundary z-measure.

Theorem. Except the equality $P_{z, z^{\prime}}=P_{z^{\prime}, z}$, the boundary $z$-measures with different parameters are mutually singular with respect to each over.

See $[\mathrm{KOV} 2]$ for a proof.

\section{ThE "UP" AND "DOWN" OPERATORS FOR THE Z-MEASURES}

Let $\operatorname{Fun}(\mathbb{Y})$ denote the algebra of all real-valued functions on $\mathbb{Y}$ with pointwise operations. We define an algebra morphism $\Lambda \rightarrow$ Fun( $\mathbb{Y})$ by specifying it on the generators $p_{k}$, as follows

$$
p_{k}(\lambda)=\sum_{i=1}^{d} a_{i}^{k}+(-1)^{k-1} \sum_{i=1}^{d} b_{i}^{k}, \quad \lambda \in \mathbb{Y}
$$

where $a_{1}, \ldots, a_{d}$ and $b_{1}, \ldots, b_{d}$ are the modified Frobenius coordinates of $\lambda$. It is readily verified that the images of the $p_{k}$ 's in Fun $(\mathbb{Y})$ are algebraically independent, so that our morphism is injective. Thus, we may view $\Lambda$ as a subalgebra of Fun( $\mathbb{Y})$. We will denote the value at $\lambda$ of the function on $\mathbb{Y}$ corresponding to an element $f \in \Lambda$ as $f(\lambda)$. 
As mentioned above, the Schur functions $s_{\mu}$ form a distinguished basis in $\Lambda$. The Schur functions are homogeneous, the degree of $s_{\mu}$ is equal to $|\mu|$, see [Ma].

There is another important basis in $\Lambda$, formed by the Frobenius-Schur functions $F S_{\mu}$. These are inhomogeneous elements such that $F S_{\mu}$ differs from $s_{\mu}$ by lower degree terms. The crucial property of the $F S_{\mu}$ 's is expressed by the formula

$$
F S_{\mu}(\lambda)=n^{\downarrow m} \frac{\operatorname{dim}(\mu, \lambda)}{\operatorname{dim} \lambda}, \quad n=|\lambda|, \quad m=|\mu|,
$$

where we use the notation

$$
n^{\downarrow m}=n(n-1) \ldots(n-m+1)
$$

and $\operatorname{dim}(\mu, \lambda)$ denotes the number of all possible chains $\mu \nearrow \ldots \nearrow \lambda$ leading from $\mu$ to $\lambda$ (equivalently, $\operatorname{dim}(\mu, \lambda)$ equals the number of standard tableaux of the skew shape $\lambda / \mu$ if $\lambda$ contains $\mu$, and 0 otherwise). In particular, $F S_{\mu}(\lambda)$ vanishes unless $\lambda$ contains $\mu$.

For more detail about the realization of symmetric functions as functions on $\mathbb{Y}$ and about the Frobenius-Schur functions, see [KO], [OO], [OlRV], [IO].

Given $f \in \Lambda$, we denote by $f_{n}$ the restriction of the function $f(\cdot)$ to $\mathbb{Y}_{n} \subset \mathbb{Y}$. It is readily checked that the subalgebra $\Lambda \subset \operatorname{Fun}(\mathbb{Y})$ separates points, which implies that for each $n$, the functions of the form $f_{n}$, with $f \in \Lambda$, exhaust the space $C\left(\mathbb{Y}_{n}\right)$.

Let $D_{n+1, n}: C\left(\mathbb{Y}_{n}\right) \rightarrow C\left(\mathbb{Y}_{n+1}\right)$ and $U_{n, n+1}: C\left(\mathbb{Y}_{n+1}\right) \rightarrow C\left(\mathbb{Y}_{n}\right)$ be the "down" and "up" operators acting on functions:

$$
\begin{gathered}
\left(D_{n+1, n} f\right)(\nu)=\sum_{\lambda \in \mathbb{Y}_{n}} p^{\downarrow}(\nu, \lambda) f(\lambda), \quad f \in C\left(\mathbb{Y}_{n}\right), \quad \nu \in \mathbb{Y}_{n+1}, \\
\left(U_{n, n+1} g\right)(\lambda)=\sum_{\nu \in \mathbb{Y}_{n+1}} p_{z, z^{\prime}}^{\uparrow}(\lambda, \nu) g(\nu), \quad g \in C\left(\mathbb{Y}_{n+1}\right), \quad \lambda \in \mathbb{Y}_{n} .
\end{gathered}
$$

Note that $U_{n, n+1}$ depends on $z$ and $z^{\prime}$ while $D_{n+1, n}$ does not.

In this section, we prove the following claim.

Theorem 4.1. (1) There exists a unique operator $\widetilde{D}: \Lambda \rightarrow \Lambda$ such that

$$
D_{n+1, n} f_{n}=\frac{1}{n+1}(\widetilde{D} f)_{n+1}, \quad \text { for all } n=0,1, \ldots \text { and all } f \in \Lambda .
$$

In the basis $\left\{F S_{\mu}\right\}$ it is given by

$$
\widetilde{D} F S_{\mu}=\left(p_{1}-|\mu|\right) F S_{\mu}, \quad \mu \in \mathbb{Y} .
$$

(2) There exists a unique operator $\widetilde{U}: \Lambda \rightarrow \Lambda$ depending on $z, z^{\prime}$, such that

$$
U_{n, n+1} f_{n+1}=\frac{1}{z z^{\prime}+n}(\widetilde{U} f)_{n}, \quad \text { for all } n=0,1, \ldots \text { and all } f \in \Lambda .
$$

In the basis $\left\{F S_{\mu}\right\}$ it is given by

$$
\widetilde{U} F S_{\mu}=\sum_{\mu_{\bullet} \nearrow \mu}(z)_{\mu / \mu_{\bullet}}\left(z^{\prime}\right)_{\mu / \mu_{\bullet}} F S_{\mu_{\bullet}}+\left(p_{1}+z z^{\prime}+|\mu|\right) F S_{\mu}, \quad \mu \in \mathbb{Y} .
$$


Proof of (1). Uniqueness follows from the fact that $\Lambda \rightarrow$ Fun( $\mathbb{Y})$ is an embedding. Let us check the required relation. Denote $m=|\mu|$. We have to prove that for any $n$ and any $\nu \in \mathbb{Y}_{n+1}$,

$$
\left(D_{n+1, n}\left(F S_{\mu}\right)_{n}\right)(\nu)=\frac{n+1-m}{n+1} F S_{\mu}(\nu)
$$

(here we have used the fact that $p_{1}(\nu)=n+1$ ).

If $n<m$ then the equality holds for trivial reasons: both sides vanish. Indeed, we have $\left(F S_{\mu}\right)_{n} \equiv 0$, the factor $n+1-m$ vanishes for $n=m-1$, and $F S_{\mu}(\nu)=0$ for $n<m-1$. Thus, we may assume $n \geq m$.

Then we have

$$
\begin{aligned}
\left(D_{n+1, n}\left(F S_{\mu}\right)_{n}\right)(\nu) & =\sum_{\nu_{\bullet} \nearrow \nu} \frac{\operatorname{dim} \nu_{\bullet}}{\operatorname{dim} \nu} F S_{\mu}\left(\nu_{\bullet}\right) \\
& =\sum_{\nu_{\bullet} \nearrow_{\nu}} \frac{\operatorname{dim} \nu_{\bullet}}{\operatorname{dim} \nu} n^{\downarrow m} \frac{\operatorname{dim}\left(\mu, \nu_{\bullet}\right)}{\operatorname{dim} \nu_{\bullet}} \quad \text { by }(4.1) \\
& =n^{\downarrow m} \sum_{\nu \bullet \nearrow \nu} \frac{\operatorname{dim}\left(\mu, \nu_{\bullet}\right)}{\operatorname{dim} \nu} \\
& =n^{\downarrow m} \frac{\operatorname{dim}(\mu, \nu)}{\operatorname{dim} \nu} \\
& =\frac{n^{\downarrow m}}{(n+1)^{\downarrow m}} F S_{\mu}(\nu) \quad \text { by }(4.1) \\
& =\frac{n+1-m}{n+1} F S_{\mu}(\nu), \quad
\end{aligned}
$$

as required.

The proof of (2) is more involved and depends on the lemma below which is essentially due to Sergei Kerov (see Okounkov [Ok]).

Let $\operatorname{Fun}_{0}(\mathbb{Y}) \subset \operatorname{Fun}(\mathbb{Y})$ be the space of functions with finite support, and let $\left\{\delta_{\lambda}\right\}$ be its natural basis: $\delta_{\lambda}(\lambda)=1$ and $\delta_{\lambda}(\nu)=0$ for $\nu \neq \lambda$. Consider the Lie algebra $\mathfrak{s l}(2, \mathbb{C})$ with its basis

$$
E=\left[\begin{array}{ll}
0 & 1 \\
0 & 0
\end{array}\right], \quad F=\left[\begin{array}{ll}
0 & 0 \\
1 & 0
\end{array}\right], \quad H=\left[\begin{array}{cc}
1 & 0 \\
0 & -1
\end{array}\right] .
$$

Lemma 4.2. For any complex $z$ and $z^{\prime}$, the following action of $E, F$, and $H$ in the basis $\left\{\delta_{\lambda}\right\}$ defines a representation of $\mathfrak{s l}(2, \mathbb{C})$ in $\operatorname{Fun}_{0}(\mathbb{Y})$

$$
E \delta_{\lambda}=\sum_{\lambda \bullet \searrow_{\lambda}}(z)_{\lambda \bullet / \lambda}\left(z^{\prime}\right)_{\lambda \bullet / \lambda} \delta_{\lambda} \bullet, \quad F \delta_{\lambda}=-\sum_{\lambda_{\bullet} \succ \lambda} \delta_{\lambda_{\bullet}}, \quad H \delta_{\lambda}=\left(z z^{\prime}+2|\lambda|\right) \delta_{\lambda} .
$$

Proof. The only nontrivial commutation relation to be checked is $[E, F]=H$. We have

$$
[E, F] \delta_{\lambda}=\sum_{\varkappa \nearrow \lambda \bullet \lambda_{\bullet} \searrow_{\lambda}}(z)_{\lambda \bullet / \lambda}\left(z^{\prime}\right)_{\lambda \bullet / \lambda} \delta_{\varkappa}-\sum_{\varkappa \searrow \lambda_{\bullet} \lambda_{\bullet} \lambda_{\lambda}}(z)_{\varkappa / \lambda_{\bullet}}\left(z^{\prime}\right)_{\varkappa / \lambda_{\bullet}} \delta_{\varkappa}
$$


The right-hand side is a linear combination of the vectors $\delta_{\varkappa}$ such that either $\varkappa=\lambda$ or $\varkappa$ is obtained from $\lambda$ by adding a box $\square_{1}$ and removing another box $\square_{2} \neq \square_{1}$. In the latter case, the coefficient of $\delta_{\varkappa}$ in each double sum equals $(z) \square_{1}\left(z^{\prime}\right) \square_{1}$, so that the total coefficient is 0 .

Examine now the coefficient of $\delta_{\lambda}$, which is equal to

$$
\sum_{\lambda \bullet \backslash \lambda}(z)_{\lambda \bullet / \lambda}\left(z^{\prime}\right)_{\lambda \bullet / \lambda}-\sum_{\lambda_{\bullet} \gamma_{\lambda}}(z)_{\lambda / \lambda_{\bullet}}\left(z^{\prime}\right)_{\lambda / \lambda}
$$

Denoting by $\left\{x_{i}\right\}$ and $\left\{y_{j}\right\}$ the contents of the boxes ${ }^{10}$ that can be added to the diagram $\lambda$ or removed from it, respectively, we write the above expression as

$$
\begin{aligned}
& \sum_{i}\left(z+x_{i}\right)\left(z^{\prime}+x_{i}\right)-\sum_{j}\left(z+y_{j}\right)\left(z^{\prime}+y_{j}\right) \\
& \quad=\left(\sum_{i} 1-\sum_{j} 1\right) z z^{\prime}+\left(\sum_{i} x_{i}-\sum_{j} y_{j}\right)\left(z+z^{\prime}\right)+\left(\sum_{i} x_{i}^{2}-\sum_{j} y_{j}^{2}\right) .
\end{aligned}
$$

As was first observed by Kerov (see his book [K, Ch. IV, $\S 1]$ ), the $x_{i}$ 's and the $y_{j}$ 's form two interlacing sequences

$$
x_{1}<y_{1}<x_{2}<\cdots<x_{k}<y_{k}<x_{k+1}
$$

such that

$$
\sum_{i} x_{i}-\sum_{j} y_{j}=0, \quad \sum_{i} x_{i}^{2}-\sum_{j} y_{j}^{2}=2|\lambda| .
$$

The easiest way to prove this is to proceed by induction on $|\lambda|$, by consecutively adding a box to the diagram.

It follows that in our expression, the coefficient of $z z^{\prime}$ equals 1 (because the number of $x$ 's is greater than the number of $y$ 's by 1 ), that of $z+z^{\prime}$ equals 0 , and the last term equals $2|\lambda|$. This completes the proof.

The next lemma is a simple observation:

Lemma 4.3. Let $\square_{k, l}$ denote the rectangular diagram with $k$ rows and $l$ columns, and $V_{k, l} \subset \mathrm{Fun}_{0}(\mathbb{Y})$ stand for the finite dimensional subspace spanned by the basis vectors $\delta_{\lambda}$ such that $\lambda \subseteq \square_{k, l}$.

If $z=k$ and $z^{\prime}=-l$ then $V_{k, l}$ is $\mathfrak{s l}(2, \mathbb{C})$-invariant and the action of $\mathfrak{s l}(2, \mathbb{C})$ in $V_{k, l}$ lifts to a representation of the group $S L(2, \mathbb{C})$.

Proof. If a diagram $\lambda$ is contained in $\square_{k, l}$ while a diagram $\lambda^{\bullet}$, such that $\lambda^{\bullet} \searrow \lambda$, is not, then the square $\lambda^{\bullet} / \lambda$ may be only one of the boxes $(1, l+1)$ or $(k+1,1)$. In the former case, $\left(z^{\prime}\right)_{\lambda \bullet / \lambda}$ vanishes, and in the latter case $(z)_{\lambda} \bullet / \lambda$ vanishes. Therefore, the coefficient of $\delta_{\lambda}$ • in the expansion of $E \delta_{\lambda}$ equals 0 . It follows that the subspace $V_{k, l}$ is $E$-invariant, and its invariance with respect to $F$ and $H$ is obvious. Thus, $V_{k, l}$ is an $\mathfrak{s l}(2, \mathbb{C})$-module. Since it has finite dimension, it generates a representation of the group $S L(2, \mathbb{C})$. Note that this representation is irreducible but we do not need this fact.

\footnotetext{
${ }^{10}$ Recall that the content of a box $(i, j)$ is defined as $j-i$.
} 
We proceed to the proof of the second claim of Theorem 4.1.

Proof of (2). As in (1), the uniqueness part of the claim is evident. The remaining (nontrivial) part of the claim means that for any $n$ and any $\lambda \in \mathbb{Y}_{n}$,

$$
\begin{aligned}
\left(z z^{\prime}+n\right)\left(U_{n, n+1}\left(F S_{\mu}\right)_{n+1}\right)(\lambda) & =\sum_{\mu_{\bullet} \nearrow^{\mu}}(z)_{\mu / \mu \bullet}\left(z^{\prime}\right)_{\mu / \mu_{\bullet}} F S_{\mu_{\bullet}}(\lambda) \\
& +\left(n+z z^{\prime}+|\mu|\right) F S_{\mu}(\lambda) .
\end{aligned}
$$

For $\mu=\varnothing, F S_{\mu}$ reduces to the constant function 1, the sum in the right-hand side disappears, and (4.2) reduces to the tautology $z z^{\prime}+n=z z^{\prime}+n$.

Assume now $|\mu|=m \geq 1$. Using the definition of $U_{n, n+1}$ and the basic formula (4.1), one can reduce (4.2) to the following combinatorial identity

$$
\begin{aligned}
\sum_{\lambda \bullet \searrow \lambda}(z)_{\lambda \bullet / \lambda}\left(z^{\prime}\right)_{\lambda \bullet / \lambda} n^{\downarrow(m-1)} \operatorname{dim}\left(\mu, \lambda^{\bullet}\right) & =\sum_{\mu_{\bullet} \nearrow_{\mu}}(z)_{\mu / \mu_{\bullet}}\left(z^{\prime}\right)_{\mu / \mu_{\bullet}} n^{\downarrow(m-1)} \operatorname{dim}\left(\mu_{\bullet}, \lambda\right) \\
& +\left(n+z z^{\prime}+m\right) n^{\downarrow m} \operatorname{dim}(\mu, \lambda)
\end{aligned}
$$

If $n<m-1$ then both sides vanish. Thus, we may assume $n \geq m-1$, so that $n^{\downarrow(m-1)} \neq 0$. Dividing by $n^{\downarrow(m-1)}$ we reduce the identity to

$$
\begin{aligned}
\sum_{\lambda \bullet \backslash \lambda}(z)_{\lambda \bullet / \lambda}\left(z^{\prime}\right)_{\lambda \bullet / \lambda} \operatorname{dim}(\mu, \lambda \bullet) & =\sum_{\mu_{\bullet} \nearrow_{\mu}}(z)_{\mu / \mu_{\bullet}}\left(z^{\prime}\right)_{\mu / \mu_{\bullet}} \operatorname{dim}\left(\mu_{\bullet}, \lambda\right) \\
& +\left(n+z z^{\prime}+m\right)(n-m+1) \operatorname{dim}(\mu, \lambda),
\end{aligned}
$$

Observe that the identity is satisfied if $\lambda$ does not contain $\mu$. Indeed, in such a case $\operatorname{dim}(\mu, \lambda)=0$, and the last summand disappears. If the set difference $\mu \backslash \lambda$ contains 2 or more boxes then no $\lambda^{\bullet}$ contains $\mu$ and no $\mu_{\bullet}$ is contained in $\lambda$, so that both sides vanish. Examine now the case when $\mu \backslash \lambda$ consists of a single square $\square$. Then the only nonzero contribution to the left-hand side comes from the summand with $\lambda^{\bullet}=\lambda \cup \square$, and the only nonzero contribution to the right-hand side comes from $\mu_{\bullet}=\mu \backslash \square$. Since $\lambda \bullet / \lambda=\mu / \mu_{\bullet}=\square$, the identity is reduced to $\operatorname{dim}\left(\mu, \lambda^{\bullet}\right)=\operatorname{dim}\left(\mu_{\bullet}, \lambda\right)$, which is obvious, because the skew diagrams $\lambda^{\bullet} / \mu$ and $\lambda / \mu_{\bullet}$ coincide.

Thus, we may assume $\mu \subseteq \lambda$. We will check the identity using Lemma 4.2. Since both sides are polynomials in $z$ and $z^{\prime}$, we may assume that $z=k$ and $z^{\prime}=-l$, where $k$ and $l$ are so large that all diagrams $\lambda \bullet$ are contained in $\square_{k, l}$.

Let us multiply the left-hand side of (4.3) by $(z)_{\lambda / \mu}\left(z^{\prime}\right)_{\lambda / \mu}$. Due to our assumptions this quantity is well defined and is nonzero. We obtain

$$
\begin{aligned}
& (z)_{\lambda / \mu}\left(z^{\prime}\right)_{\lambda / \mu} \sum_{\lambda \bullet \searrow \lambda}(z)_{\lambda \bullet / \lambda}\left(z^{\prime}\right)_{\lambda \bullet / \lambda} \operatorname{dim}\left(\mu, \lambda^{\bullet}\right)=\sum_{\lambda \bullet \backslash \lambda}(z)_{\lambda \bullet / \mu}\left(z^{\prime}\right)_{\lambda \bullet / \mu} \operatorname{dim}\left(\mu, \lambda^{\bullet}\right) \\
& =\sum_{\lambda \bullet \searrow \lambda}\left(E^{n-m+1} \delta_{\mu}, \delta_{\lambda} \bullet\right)=(n+1-m) ! \sum_{\lambda \bullet \searrow \lambda}\left(e^{E} \delta_{\mu}, \delta_{\lambda} \bullet\right) \\
& =(n+1-m) !\left(e^{E} \delta_{\mu}, \sum_{\lambda \bullet \searrow_{\lambda}} \delta_{\lambda} \bullet\right),
\end{aligned}
$$

where all operators act in the finite-dimensional subspace $V_{k, l}$ described in Lemma 4.3 , and $(\cdot, \cdot)$ is the natural inner product inherited from $\ell^{2}(\mathbb{Y})$. 
Since the operator $\delta_{\lambda} \mapsto \sum_{\lambda} \bullet \searrow_{\lambda} \delta_{\lambda} \bullet$ is adjoint to $-F$, our expression can be rewritten simply as

$$
-(n+1-m) !\left(F e^{E} \delta_{\mu}, \delta_{\lambda}\right) .
$$

A simple computation in $S L(2, \mathbb{C})$ shows that

$$
-F e^{E}=-e^{E}\left(e^{-E} F e^{E}\right)=e^{E}(-F+E+H),
$$

and due to the last claim of Lemma 4.3 we may interpret the above identity as a relation between operators in $V_{k, l}$. Then we obtain

$$
\begin{gathered}
-\left(F e^{E} \delta_{\mu}, \delta_{\lambda}\right)=-\left(e^{E} F \delta_{\mu}, \delta_{\lambda}\right)+\left(e^{E} H \delta_{\mu}, \delta_{\lambda}\right)+\left(e^{E} E \delta_{\mu}, \delta_{\lambda}\right) \\
=\sum_{\mu_{\bullet} \nearrow_{\mu}}\left(e^{E} \delta_{\mu_{\bullet}}, \delta_{\lambda}\right)+\left(z z^{\prime}+2 m\right)\left(e^{E} \delta_{\mu}, \delta_{\lambda}\right)+\left(E e^{E} \delta_{\mu}, \delta_{\lambda}\right)
\end{gathered}
$$

It remains to check that multiplying $(4.4)$ by $(n-m+1)$ ! gives the right-hand side of (4.3) multiplied by $(z)_{\lambda / \mu}\left(z^{\prime}\right)_{\lambda / \mu}$. The expression (4.4) comprises three terms.

The first term gives

$$
\begin{gathered}
(n-m+1) ! \sum_{\mu_{\bullet} \nearrow_{\mu}}\left(e^{E} \delta_{\mu_{\bullet}}, \delta_{\lambda}\right)=\sum_{\mu_{\bullet} \nearrow_{\mu}}(z)_{\lambda / \mu_{\bullet}}\left(z^{\prime}\right)_{\lambda / \mu_{\bullet}} \operatorname{dim}\left(\mu_{\bullet}, \lambda\right) \\
=(z)_{\lambda / \mu}\left(z^{\prime}\right)_{\lambda / \mu} \sum_{\mu_{\bullet} \nearrow_{\mu}}(z)_{\mu / \mu_{\bullet}}\left(z^{\prime}\right)_{\mu / \mu_{\bullet}} \operatorname{dim}\left(\mu_{\bullet}, \lambda\right) .
\end{gathered}
$$

Next, the second term gives

$(n-m+1) !\left(z z^{\prime}+2 m\right)\left(e^{E} \delta_{\mu}, \delta_{\lambda}\right)=(z)_{\lambda / \mu}\left(z^{\prime}\right)_{\lambda / \mu}\left(z z^{\prime}+2 m\right)(n-m+1) \operatorname{dim}(\mu, \lambda)$, the third term gives

$$
(n-m+1) !\left(E e^{E} \delta_{\mu}, \delta_{\lambda}\right)=(z)_{\lambda / \mu}\left(z^{\prime}\right)_{\lambda / \mu}(n-m+1)(n-m) \operatorname{dim}(\mu, \lambda),
$$

and their sum equals

$$
(z)_{\lambda / \mu}\left(z^{\prime}\right)_{\lambda / \mu}\left(z z^{\prime}+n+m\right)(n-m+1) \operatorname{dim}(\mu, \lambda) .
$$

We see that the sum of (4.5) and (4.6) is indeed equal to the right-hand side of (4.3) multiplied by $(z)_{\lambda / \mu}\left(z^{\prime}\right)_{\lambda / \mu}$.

\section{Convergence of Markov semigroups}

Fix arbitrary parameters $\left(z, z^{\prime}\right)$ of principal or complementary series. Let $\left\{M_{n}\right\}=$ $\left\{M_{z, z^{\prime}}^{(n)}\right\}$ be the corresponding coherent family of probability measures on $\mathbb{Y}$. According to the general formalism of $\$ 1$, we form, for each $n=1,2, \ldots$, the $n$th level up-down Markov chain on the set $\mathbb{Y}_{n}$ of Young diagrams with $n$ boxes. Since the move "up" consists in appending a box to a Young diagram, while the move "down" consists in removing a box, any nontrivial change of our up-down chain under one step reduces to moving one of the boxes of a Young diagram to a new position.

The measure $M_{n}$ is an invariant measure of the chain. It is readily seen that all the states are communicating, so that $M_{n}$ is a unique invariant probability measure.

As explained in $\S 3$, we consider the embeddings $\iota_{n}: \mathbb{Y}_{n} \rightarrow \Omega$ determined by the normalized modified Frobenius coordinates. Let $\pi_{n}$ be the corresponding linear $\operatorname{map} C(\Omega) \rightarrow C\left(\mathbb{Y}_{n}\right)$.

As the space $\mathcal{F} \subset C(\Omega)$ we take the algebra $\Lambda^{\circ}$. It is dense in $C(\Omega)$ by virtue of [KOO, Lemma 5.3]. The filtration in $\Lambda^{\circ}$ is inherited from $\Lambda$.

The two limit relations in the claim below are understood in the sense of $\S 1$. 
Theorem 5.1. With these data, all the assumptions of Proposition 1.4 are satisfied provided that the scaling of time is determined by the factors $\varepsilon_{n} \sim n^{-2}$. Thus, denoting by $T_{n}$ the Markov operator of the nth level Markov chain, we have convergence to a conservative Markov semigroup $\{T(t)\}$ in the Banach space $C(\Omega)$, as in (1.4):

$$
\lim _{n \rightarrow \infty} T^{\left[n^{2} t\right]} \pi_{n}(f)=T(t) f
$$

for any fixed $f \in C(\Omega)$ and all $t \geq 0$, uniformly on bounded intervals. Furthermore, the generator of the limit semigroup $\{T(t)\}$ is the closure of the operator $A$ with domain $\Lambda^{\circ} \subset C(\Omega)$, defined by

$$
\text { Af }=\lim _{n \rightarrow \infty} n^{2}\left(T_{n}-\mathbf{1}\right) f, \quad f \in \Lambda^{\circ} .
$$

Proof. By virtue of Proposition 1.4, it suffices to check assumptions (A1)-(A5) stated before the formulation of the proposition.

The fulfilment of assumptions (A1), (A2), and (A5) is obvious.

We proceed to verifying assumption (A3).

Recall that the Markov operator $T_{n}: C\left(\mathbb{Y}_{n}\right) \rightarrow C\left(\mathbb{Y}_{n}\right)$ is defined as the composition $U_{n, n+1} \circ D_{n+1, n}$ of the up and down operators. As shown in $\S 4$, the latter operators are implemented by certain operators in $\Lambda$. Here we interpret $\Lambda$ as a subalgebra in $\operatorname{Fun}(\mathbb{Y})$ and consider the restriction map $\operatorname{Fun}(\mathbb{Y}) \rightarrow C\left(\mathbb{Y}_{n}\right)$ turning elements $f \in \Lambda$ to functions $f_{n}$ on $\mathbb{Y}_{n}$.

Lemma 5.2. Let $\mu$ be a Young diagram and $m=|\mu|$. The operator $T_{n}-\mathbf{1}$ acts on $\left(F S_{\mu}\right)_{n}$ as follows

$$
\begin{aligned}
& \left(T_{n}-\mathbf{1}\right)\left(F S_{\mu}\right)_{n}=-\frac{m\left(m-1+z z^{\prime}\right)}{(n+1)\left(z z^{\prime}+n\right)}\left(F S_{\mu}\right)_{n} \\
& \quad+\frac{n+1-m}{(n+1)\left(z z^{\prime}+n\right)} \sum_{\mu_{\bullet} \nearrow_{\mu}}(z)_{\mu_{\mu} \mu_{\bullet}}\left(z^{\prime}\right)_{\mu / \mu_{\bullet}}\left(F S_{\mu_{\bullet}}\right)_{n} .
\end{aligned}
$$

Proof. This follows directly from the computation of $\S 4$. Indeed, we have

$$
\begin{gathered}
T_{n}\left(F S_{\mu}\right)_{n}=U_{n, n+1} D_{n+1, n}\left(F S_{\mu}\right)_{n}, \\
D_{n+1, n}\left(F S_{\mu}\right)_{n}=\frac{n+1-m}{n+1}\left(F S_{\mu}\right)_{n+1}, \\
U_{n, n+1}\left(F S_{\mu}\right)_{n+1}=\frac{1}{z z^{\prime}+n}\left(\sum_{\mu \bullet \nearrow \mu}(z)_{\mu / \mu_{\bullet}}\left(z^{\prime}\right)_{\mu / \mu_{\bullet}}\left(F S_{\mu_{\bullet}}\right)_{n}+\left(n+z z^{\prime}+m\right)\left(F S_{\mu}\right)_{n}\right),
\end{gathered}
$$

which implies the desired expression. Note that in the last equality we used the fact that $\left(p_{1} F S_{\mu}\right)_{n}=n\left(F S_{\mu}\right)_{n}$.

Corollary 5.3. $T_{n}$ preserves the filtration in $\mathcal{F}$.

Let us verify assumption (A4) with $\varepsilon_{n}=n^{-2}$ :

Consider the map $\Lambda \rightarrow C\left(\mathbb{Y}_{n}\right)$, defined as restriction to $\iota_{n}\left(\mathbb{Y}_{n}\right) \subset \Omega$. We denote it as $f \mapsto f_{[n]}$ (it should not be confused with the map $f \mapsto f_{n}$ introduced in the 
beginning of $\S 4 !)$. By the very definition, if $\lambda=\left(a_{1}, \ldots, a_{d} \mid b_{1}, \ldots, b_{d}\right) \in \mathbb{Y}_{n}$ (the modified Frobenius coordinates) then

$$
f_{[n]}(\lambda)=f\left(\frac{a_{1}}{n}, \ldots, \frac{a_{d}}{n}, 0,0, \ldots ; \frac{b_{1}}{n}, \ldots, \frac{b_{d}}{n}, 0,0, \ldots\right), \quad f \in \Lambda .
$$

In the notation of $\S 1$,

$$
f_{[n]}=\pi_{n}\left(f^{\circ}\right), \quad f \in \Lambda .
$$

Let $G: \Lambda \rightarrow \Lambda$ denote the operator acting on the $m$ th homogeneous component of $\Lambda$ as multiplication by $m(m=0,1,2, \ldots)$. According to this definition, we denote by $s^{G}$, where $s \neq 0$, the automorphism of the algebra $\Lambda$ that reduces to multiplication by $s^{m}$ on the homogeneous component of degree $m$. Then we have

$$
f_{n}(\lambda)=\left(n^{G} f\right)_{[n]}(\lambda), \quad \lambda \in \mathbb{Y}_{n} .
$$

Indeed, it suffices to check this formula for $f=p_{k}$, and then it follows from the very definition of the embedding $\Lambda \rightarrow$ Fun( $\mathbb{Y}$ ), see the beginning of $\S 4$.

Lemma 5.4. There exists a linear operator $A_{z, z^{\prime}}$ in $\mathcal{F}=\Lambda^{\circ}$ which is the limit of the operators $n^{2}\left(T_{n}-\mathbf{1}\right)$ as $n \rightarrow \infty$. Specifically,

$$
A_{z, z^{\prime}} s_{\mu}^{\circ}=-m\left(m-1+z z^{\prime}\right) s_{\mu}^{\circ}+\sum_{\mu_{\bullet} \nearrow \mu}(z)_{\mu / \mu_{\bullet}}\left(z^{\prime}\right)_{\mu / \mu_{\bullet}} s_{\mu_{\bullet}}^{\circ}, \quad \mu \in \mathbb{Y}, \quad m:=|\mu| .
$$

Proof. Rewrite the claim of Lemma 5.2 where we substitute $\left(F S_{\mu}\right)_{n}=\left(n^{G} F S_{\mu}\right)_{[n]}$ and $\left(F S_{\mu_{\bullet}}\right)_{n}=\left(n^{G} F S_{\mu_{\bullet}}\right)_{[n]}$ :

$$
\begin{aligned}
\left(T_{n}-\mathbf{1}\right)\left(n^{G} F S_{\mu}\right)_{[n]}=- & \frac{m\left(m-1+z z^{\prime}\right)}{(n+1)\left(z z^{\prime}+n\right)}\left(n^{G} F S_{\mu}\right)_{[n]} \\
& +\frac{n+1-m}{(n+1)\left(z z^{\prime}+n\right)} \sum_{\mu_{\bullet} \nearrow_{\mu}}(z)_{\mu / \mu \bullet}\left(z^{\prime}\right)_{\mu / \mu \bullet}\left(n^{G} F S_{\mu_{\bullet}}\right)_{[n]} .
\end{aligned}
$$

Multiply both sides by $n^{2} \cdot n^{-m}$ and observe that

$$
\lim _{n \rightarrow \infty} n^{-m} n^{G} F S_{\mu}=s_{\mu}, \quad \lim _{n \rightarrow \infty} n^{-m+1} n^{G} F S_{\mu_{\bullet}}=s_{\mu_{\bullet}},
$$

because $F S_{\mu}$ and $s_{\mu}$, as well as $F S_{\mu_{\bullet}}$ and $s_{\mu_{\bullet}}$, differ in lower order terms only. This implies the claim of the lemma.

Note that for $\mu=\varnothing$, the sum in (5.1) disappears and $m$ vanishes, so that $A_{z, z^{\prime}}$ sends $s_{\varnothing}^{\circ}=1$ to 0 , as it should be.

This concludes the proof of Theorem 5.1.

Looking at formula (5.1), it is not obvious that it defines an operator in $\Lambda^{\circ}$, because the elements $s_{\mu}^{\circ}$ are not linearly independent. Of course, correctness of (5.1) follows from the computation in the proof of Lemma 5.4. On the other hand, this also can be proved directly: 
Proposition 5.5. The operator $B_{z, z^{\prime}}: \Lambda \rightarrow \Lambda$ determined in the basis of Schur functions by the expression

$$
B_{z, z^{\prime}} s_{\mu}=-|\mu|\left(|\mu|-1+z z^{\prime}\right) s_{\mu}+p_{1} \sum_{\mu_{\bullet}{ }_{\mu}}(z)_{\mu / \mu}\left(z^{\prime}\right)_{\mu / \mu_{\bullet}} s_{\mu}
$$

preserves the principal ideal generated by $p_{1}-1$, and the reduction of $B_{z, z^{\prime}}$ modulo this ideal coincides with the expression (5.1).

Proof. The second claim is obvious from the comparison of (5.2) with (5.1); note that the prefactor $p_{1}$ in front of the sum in (5.2) will disappear after the reduction. Note also that the operator $B$ preserves the grading in $\Lambda$.

To prove the first claim it suffices to check that $B$ commutes with the operator of multiplication by $p_{1}$.

We use the representation of the Lie algebra $\mathfrak{s l}(2, \mathbb{C})$ in the $\operatorname{space}^{\mathrm{Fun}}(\mathbb{Y})$ defined in Lemma 4.2. Let $H^{*}, E^{*}$, and $F^{*}$ be the adjoint operators to $H, E$, and $F$, respectively. We interpret them again as operators in $\operatorname{Fun}_{0}(\mathbb{Y})$, given by adjoint matrices in the basis $\left\{\delta_{\mu}\right\}$ (equivalently, by transposed matrices, because all the matrices in questions have real entries). Note that $H^{*}=H$.

Now identify $\operatorname{Fun}_{0}(\mathbb{Y})$ and $\Lambda$ (as vector spaces) via the correspondence $\delta_{\mu} \leftrightarrow s_{\mu}$. Then we may interpret $H^{*}=H, E^{*}$, and $F^{*}$ as operators in $\Lambda$. From the definition of $F$ and the well-known identity

$$
p_{1} s_{\lambda}=\sum_{\lambda \bullet \searrow \lambda} s_{\lambda} \bullet
$$

it follows that the operator of multiplication by $p_{1}$ equals $-F^{*}$. Next, the operator

$$
s_{\mu} \mapsto \sum_{\mu_{\bullet} \nearrow \mu}(z)_{\mu / \mu_{\bullet}}\left(z^{\prime}\right)_{\mu / \mu_{\bullet}} s_{\mu_{\bullet}}
$$

equals $E^{*}$. Finally, recall the operator $G$ introduced just before Lemma 5.4 and note that

$$
G: s_{\mu} \mapsto|\mu| s_{\mu}
$$

Therefore, one can write

$$
B_{z, z^{\prime}}=-G(G-1+\theta)-F^{*} E^{*}, \quad \theta:=z z^{\prime} .
$$

We have to check that this operator commutes with $-F^{*}$, which is equivalent to

$$
[G(G-1+\theta)+E F, F]=0 .
$$

Observe that $H=2 G+\theta$. Therefore

$$
G(G-1+\theta)+E F=\frac{1}{4} H^{2}-\frac{1}{2} H+E F-\frac{1}{4} \theta^{2}+\frac{1}{2} \theta .
$$

It is readily checked that this expression gives a central element in the universal enveloping algebra of $\mathfrak{s l}(2, \mathbb{C})$. 


\section{The Pre-Generator $A_{z, z^{\prime}}$ AS a Differential operator}

Recall (see $\S \S 3.4-3.5)$ that the algebra $\Lambda^{\circ}$ can be identified with the polynomial ring $\mathbb{R}\left[q_{1}, q_{2}, \ldots\right]$. Note that any linear operator in the vector space of polynomials with countably many indeterminates can be written as a differential operator, that is, as an infinite sum of differential monomials with polynomial coefficients. Our aim here is to write in this form the operator $A_{z, z^{\prime}}: \Lambda^{\circ} \rightarrow \Lambda^{\circ}$ defined by (5.1).

Theorem 6.1. In the moment coordinates $q_{1}=p_{2}^{\circ}, q_{2}=p_{3}^{\circ}, \ldots$, the operator $A_{z, z^{\prime}}$ defined by (5.1) can be written as the differential operator

$$
\begin{aligned}
& A_{z, z^{\prime}}=\sum_{i, j=1}^{\infty}(i+1)(j+1)\left(q_{i+j}-q_{i} q_{j}\right) \frac{\partial^{2}}{\partial q_{i} \partial q_{j}} \\
&-z z^{\prime} \sum_{i=1}^{\infty}(i+1) q_{i} \frac{\partial}{\partial q_{i}}+\left(z+z^{\prime}\right) \sum_{i=1}^{\infty}(i+1) q_{i-1} \frac{\partial}{\partial q_{i}} \\
& \quad+\sum_{i, j=0}^{\infty}(i+j+3) q_{i} q_{j} \frac{\partial}{\partial q_{i+j+2}}-\sum_{i=1}^{\infty}(i+1) i q_{i} \frac{\partial}{\partial q_{i}}
\end{aligned}
$$

where, by agreement, $q_{0}=1$.

Proof. We will show that the operator $B_{z, z^{\prime}}: \Lambda \rightarrow \Lambda$ (see (5.2)) can be written as the following differential operator in the indeterminates $p_{1}, p_{2}, \ldots$ :

$$
\begin{aligned}
B_{z, z^{\prime}}=\sum_{i, j=2}^{\infty} i j\left(p_{1} p_{i+j-1}-p_{i} p_{j}\right) \frac{\partial^{2}}{\partial p_{i} \partial p_{j}} & \\
- & z z^{\prime} \sum_{i=2}^{\infty} i p_{i} \frac{\partial}{\partial p_{i}}+\left(z+z^{\prime}\right) \sum_{i=2}^{\infty} i p_{1} p_{i-1} \frac{\partial}{\partial p_{i}} \\
& +\sum_{i, j=1}^{\infty}(i+j+1) p_{1} p_{i} p_{j} \frac{\partial}{\partial p_{i+j+1}}-\sum_{i=2}^{\infty} i(i-1) p_{i} \frac{\partial}{\partial p_{i}}
\end{aligned}
$$

Note that (6.2) does not involve $\partial / \partial p_{1}$, so that we can reduce (6.2) modulo the relation $p_{1}=1$, by sending $p_{i}$ to $p_{i}^{\circ}=q_{i-1}$, with the understanding that $p_{1}^{\circ}=q_{0}=$ 1. By virtue of Proposition 5.5 this will give us (6.1).

The main difficulty is to handle the sum in the right-hand side of (5.2), that is, the operator in $\Lambda$ defined by

$$
s_{\mu} \mapsto \sum_{\mu_{\bullet} \nearrow \mu}(z)_{\mu / \mu_{\bullet}}\left(z^{\prime}\right)_{\mu / \mu_{\bullet}} s_{\mu_{\bullet}} .
$$

Given a box $\square$ with the row coordinate $i$ and the column coordinate $j$, we denote by $c(\square)$ the content $j-i$. The latter operator can be written as a linear combination of three operators,

$$
C_{2}^{\prime}+\left(z+z^{\prime}\right) C_{1}^{\prime}+z z^{\prime} C_{0}^{\prime},
$$

where by $C_{k}^{\prime}: \Lambda \rightarrow \Lambda$ we denote the operator

$$
C_{k}^{\prime}: s_{\mu} \mapsto \sum_{\mu_{\bullet} \nearrow^{\mu}}\left(c\left(\mu / \mu_{\bullet}\right)\right)^{k} s_{\mu_{\bullet}}, \quad k=0,1,2, \ldots
$$


In this notation the operator $B_{z, z^{\prime}}$ is written as follows

$$
B_{z, z^{\prime}}=-G\left(G-\mathbf{1}+z z^{\prime} \mathbf{1}\right)+p_{1}\left(C_{2}^{\prime}+\left(z+z^{\prime}\right) C_{1}^{\prime}+z z^{\prime} C_{0}^{\prime}\right) .
$$

By the very definition of $G$ we have

$$
G=\sum_{i=1}^{\infty} i p_{i} \frac{\partial}{\partial p_{i}}
$$

The operator $C_{0}^{\prime}$ is also easy to write:

$$
C_{0}^{\prime}=\partial / \partial p_{1} .
$$

Indeed, to see this, one can use the fact that $\partial / \partial p_{1}$ is adjoint to the operator of multiplication by $p_{1}$, which has the form

$$
s_{\mu} \mapsto \sum_{\mu \bullet \searrow \mu} s_{\mu} \bullet
$$

The operator $C_{1}^{\prime}$ and especially the operator $C_{2}^{\prime}$ are more involved.

To handle them it is convenient to introduce auxiliary operators $C_{k}: \Lambda \rightarrow \Lambda$ by

$$
C_{k}: s_{\mu} \mapsto\left(\sum_{\square \in \mu}(c(\square))^{k}\right) s_{\mu},
$$

summed over all boxes contained in $\mu$, and observe that $C_{0}^{\prime}=\partial / \partial p_{1}$ implies

$$
C_{k}^{\prime}=\left[\frac{\partial}{\partial p_{1}}, C_{k}\right] \text {. }
$$

We will employ the following results proved by Lascoux and Thibon in [LT, Proposition 3.3]:

Lemma 6.2. Let $t$ and $u$ be formal variables. The exponential generating series for the operators $C_{k}$ has the form

$$
\sum_{k=1}^{\infty} C_{k} \frac{t^{k}}{k !}=\frac{V_{0}-1}{\left(e^{t}-1\right)\left(1-e^{-t}\right)}-G,
$$

where $V_{0}$ is the constant term of the "vertex operator"

$$
\begin{aligned}
V(u)=\sum_{m=-\infty}^{+\infty} V_{m} u^{-m} & \\
& :=\exp \left(\sum_{k=1}^{\infty}\left(e^{k t}-1\right) \frac{u^{k}}{k} p_{k}\right) \exp \left(\sum_{k=1}^{\infty}\left(1-e^{-k t}\right) u^{-k} \frac{\partial}{\partial p_{k}}\right) .
\end{aligned}
$$

Moreover, the following commutation relations hold:

$$
\left[V_{m}, k \frac{\partial}{\partial p_{k}}\right]=\left(1-e^{k t}\right) V_{m+k} .
$$

In particular,

$$
\left[\frac{\partial}{\partial p_{1}}, V_{0}\right]=\left(e^{t}-1\right) V_{1}
$$

From Lemma 6.2 we deduce explicit expressions for the operators $C_{1}^{\prime}$ and $C_{2}^{\prime}$ : 
Lemma 6.3. We have

$$
\begin{gathered}
C_{1}^{\prime}=\sum_{i=1}^{\infty}(i+1) p_{i} \frac{\partial}{\partial p_{i+1}} \\
C_{2}^{\prime}=\sum_{i, j=1}^{\infty} i j p_{i+j-1} \frac{\partial}{\partial p_{i}} \frac{\partial}{\partial p_{j}}+\sum_{i, j=1}^{\infty}(i+j+1) p_{i} p_{j} \frac{\partial}{\partial p_{i+j+1}} .
\end{gathered}
$$

Note that this particular result can also be obtained by the more elementary approach used in [FW].

Proof of Lemma 6.3. From Lemma 6.2 we get

$$
\left[\frac{\partial}{\partial p_{1}}, C_{k}\right]=\text { coefficient of } t^{k} u^{-1} \text { in } k ! \frac{V(u)}{1-e^{-t}}, \quad k=1,2, \ldots
$$

Next, write

$$
V(u)=\exp \left(\sum_{r=1}^{\infty} a_{r} t^{r}\right) \exp \left(\sum_{r=1}^{\infty} b_{r} t^{r}\right)
$$

where

$$
a_{r}=\sum_{k=1}^{\infty} \frac{k^{r} u^{k}}{r ! k} p_{k}, \quad b_{r}=(-1)^{r-1} \sum_{k=1}^{\infty} \frac{k^{r} u^{-k}}{r !} \frac{\partial}{\partial p_{k}} .
$$

Then

$$
\begin{aligned}
C_{1}^{\prime}=\left[\frac{\partial}{\partial p_{1}}, C_{1}\right]=\text { the coefficient of } t^{2} u^{-1} \text { in } \\
\frac{\frac{\exp \left(a_{1} t+a_{2} t^{2}+\ldots\right) \exp \left(b_{1} t+b_{2} t^{2}+\ldots\right)}{1-\frac{1}{2} t+\frac{1}{6} t^{2}+\ldots}}{}
\end{aligned}
$$

and

$$
\begin{aligned}
C_{2}^{\prime}=\left[\frac{\partial}{\partial p_{1}}, C_{2}\right]=\text { the coefficient of } t^{3} u^{-1} \text { in } \\
2 \cdot \frac{\exp \left(a_{1} t+a_{2} t^{2}+a_{3} t^{3}+\ldots\right) \exp \left(b_{1} t+b_{2} t^{2}+b_{3} t^{3}+\ldots\right)}{1-\frac{1}{2} t+\frac{1}{6} t^{2}-\frac{1}{24} t^{3}+\ldots},
\end{aligned}
$$

where the dependence in $u$ is hidden in (6.8). First, we compute the coefficients of $t^{2}$ and $t^{3}$ in (6.9) and (6.10), respectively.

The coefficient of $t^{2}$ in (6.9) equals

$$
a_{2}+\frac{1}{2} a_{1}^{2}+a_{1} b_{1}+\frac{1}{2} a_{1}+b_{2}+\frac{1}{2} b_{1}^{2}+\frac{1}{2} b_{1}+\frac{1}{12} .
$$

From (6.8) it immediately follows that a nonzero contribution to the term with $u^{-1}$ can come from $a_{1} b_{1}, b_{2}$, and $\frac{1}{2} b_{1}$ only. It turns out that the total contribution of $b_{2}+\frac{1}{2} b_{1}$ equals 0 . This gives (6.6). 
Likewise, the coefficient of $t^{3}$ in (6.10) equals

$$
\begin{aligned}
2 a_{3}+ & a_{2}+2 a_{1} a_{2}+2 a_{2} b_{1}+\frac{1}{2} a_{1}^{2}+\frac{1}{3} a_{1}^{3}+a_{1}^{2} b_{1}+2 a_{1} b_{2}+a_{1} b_{1} \\
& +a_{1} b_{1}^{2}+\frac{1}{6} a_{1}+2 b_{3}+b_{2}+2 b_{1} b_{2}+\frac{1}{2} b_{1}^{2}+\frac{1}{3} b_{1}^{3}+\frac{1}{6} b_{1}
\end{aligned}
$$

and a nonzero contribution to the term with $u^{-1}$ can come from

$$
2 a_{2} b_{1}+a_{1}^{2} b_{1}+2 a_{1} b_{2}+a_{1} b_{1}+a_{1} b_{1}^{2}+2 b_{3}+b_{2}+\frac{1}{6} b_{1}
$$

only. It turns out that the total contribution of $a_{1} b_{1}+2 a_{2} b_{1}+2 a_{1} b_{2}$ equals 0 , and the same holds for $2 b_{3}+b_{2}+\frac{1}{6} b_{1}$. Finally, we get (6.7).

Substituting the expressions (6.4), (6.5), (6.6), and (6.7) into (6.3) we get after cancellations the desired expression (6.2).

Let $\bar{A}_{z, z^{\prime}}$ denote the closure of the operator $A_{z, z^{\prime}}$ in $C(\Omega)$ (recall that the closure exists according to Proposition $1.4(1))$. The next result will be used in the proof of Theorem 7.1 below.

Corollary 6.4. Let $f(\omega)$ be a smooth cylinder function in the moment coordinates, that is, $f(\omega)=g\left(q_{1}(\omega), \ldots, q_{m}(\omega)\right)$ for a certain $m=1,2, \ldots$ and a certain smooth function $g\left(q_{1}, \ldots, q_{m}\right)$ in $m$ real variables, in a neighborhood of $[-1,1]^{m}$. Then $f$ enters the domain of $\bar{A}_{z, z^{\prime}}$. Moreover, $\bar{A}_{z, z^{\prime}} f$ is also a cylinder function, which can be obtained via application of the suitably truncated differential expression (6.1) to the function $g$.

Proof. First of all, note that here "truncation" means that we keep in (6.1) only terms not containing derivatives $\partial / \partial q_{i}$ with $i>m$. It is worth noting that the resulting cylinder function depends on the larger number of variables, $2 m$, because of the presence of the variables $q_{k+l}$ in (6.1). However, this does not cause problems.

To prove the claim of the proposition we observe that the function $g\left(q_{1}, \ldots, q_{m}\right)$, together with its partial derivatives of up to second order, can be approximated by a sequence $\left\{g_{n}\left(q_{1}, \ldots, q_{m}\right)\right\}$ of polynomials, uniformly on the cube $[-1,1]^{m} \subset \mathbb{R}^{m}$. Let $\left[A_{z, z^{\prime}}\right]$ stand for the truncated differential operator as explained above. The application of $\bar{A}_{z, z^{\prime}}$ to the function $\omega \mapsto g_{n}\left(q_{1}(\omega), \ldots, q_{m}(\omega)\right)$ is reduced to the application of $\left[A_{z, z^{\prime}}\right]$ to $g_{n}$. Since $g_{n} \rightarrow g$ and $\left[A_{z, z^{\prime}}\right] g_{n} \rightarrow\left[A_{z, z^{\prime}}\right] g$ uniformly on the cube $[-1,1]^{2 m}$, we see that $f$ belongs to the domain of $\bar{A}_{z, z^{\prime}}$ and $\bar{A}_{z, z^{\prime}} f$ is given by the cylinder function $\left[A_{z, z^{\prime}}\right] g$.

\section{THE LIMIT PROCESS}

The theorems of this section are almost direct consequences of the results established in sections 1, 5, and 6. Note that application of Propositions 1.6 and 1.7 is justified, because their hypothesis is satisfied due to the theorem of $§ 3.6$.

Theorem 7.1. The Markov semigroup $\{T(t)\}$ in $C(\Omega)$ constructed in $\S 5$ gives rise to a diffusion process $\boldsymbol{\omega}_{z, z^{\prime}}(t)$ in $\Omega$.

By a diffusion process we mean a strong Markov process (which can start from any point or any probability distribution) with continuous sample paths. 
Proof. Once the existence of a Markov semigroup $\{T(t)\}$ is established (Theorem 5.1 ), the existence of the corresponding strong Markov process is guaranteed by a general theorem stated above as Proposition 1.5. It remains to prove that the sample paths are continuous almost surely.

Since our semigroup acts in the space of continuous functions on a compact space, the application of Riesz's theorem implies the existence of a transition function (see, e.g., [L, §7.7, Thm. 1]). The continuity property holds if the transition function obeys the Dynkin-Kinney condition ([W, §10.3] or [EK2, Ch. 4, (2.35)]). This condition in turn holds if for any point $\omega \in \Omega$ and any its neighborhood $U \ni \omega$ one can find a function $f \in C(\Omega)$ with the following properties (see [EK2, Ch. 4, Remark 2.10]):

(1) $f$ is contained in $D\left(\bar{A}_{z, z^{\prime}}\right)$, the domain of the generator $\bar{A}_{z, z^{\prime}}$ of the semigroup;

(2) $\bar{A}_{z, z^{\prime}} f(\omega)=0$;

(3) $\|f\|=f(\omega)$ and the supremum of $f$ outside $U$ is strictly less than $f(\omega)$.

Such functions can be built using Corollary 6.4. Indeed, take $m$ so large that one can find inside $U$ a neighborhood of the form

$$
\left\{\omega^{\prime} \in \Omega: \quad\left|q_{i}\left(\omega^{\prime}\right)-q_{i}(\omega)\right|<\varepsilon, \quad 1 \leq i \leq m\right\} .
$$

Then take as $f$ a cylinder function as in Corollary 6.4, where $g$ equals 1 in a very small neighborhood of the point $\left(q_{1}(\omega), \ldots, q_{m}(\omega)\right) \in[-1,1]^{m}$ and then rapidly abates to 0. Since the differential operator (6.1) does not have a constant term, $\bar{A}_{z, z^{\prime}} f$ vanishes about $\omega$.

Let $P_{z, z^{\prime}}$ be the boundary measure on $\Omega$ corresponding to the coherent system with parameters $\left(z, z^{\prime}\right)$, and consider the inner product in $\Lambda^{\circ} \subset C(\Omega)$ given by

$$
(f, g)_{z, z^{\prime}}=\langle f \cdot g\rangle_{P_{z, z^{\prime}}}:=\int_{\Omega} f(\omega) g(\omega) P_{z, z^{\prime}}(d \omega) .
$$

Theorem 7.2. (1) The space $\Lambda^{\circ}$ can be decomposed into a direct sum of eigenspaces of the pre-generator $A_{z, z^{\prime}}$, and this decomposition is orthogonal with respect to the above inner product.

(2) The spectrum of $A_{z, z^{\prime}}$ is $\{0\} \cup\left\{-\sigma_{m}: m=2,3, \ldots\right\}$ where

$$
\sigma_{m}=m\left(m-1+z z^{\prime}\right), \quad m=2,3, \ldots
$$

(3) The eigenvalue 0 is simple, and the multiplicity of $-\sigma_{m}$ equals the number of partitions of $m$ without parts equal to 1 .

Proof. (1) This is a fact of linear algebra because the pre-generator $A_{z, z^{\prime}}$ is symmetric (Proposition 1.7) and preserves the filtration of $\Lambda^{\circ}$.

(2) Let $I$ denote the principal ideal in $\Lambda$ generated by $p_{1}-1$. We have

$$
\Lambda=\mathbb{R}\left[p_{1}, p_{2}, p_{3}, \ldots\right]=\mathbb{R}\left[p_{2}, p_{3}, \ldots\right] \oplus I
$$

so that we may identify $\Lambda^{\circ}$ with $\mathbb{R}\left[p_{2}, p_{3}, \ldots\right]$. It follows from (5.2) that for any homogeneous element $f \in \Lambda$ of degree $m$,

$$
B_{z, z^{\prime}} f=-m\left(m-1+z z^{\prime}\right) f+g+h, \quad \text { where } g \in I \text { and } \operatorname{deg} h<m .
$$


In particular, this is true for any monomial $p_{2}^{m_{2}} p_{3}^{m_{3}} \ldots$ from $\mathbb{R}\left[p_{2}, p_{3}, \ldots\right]$. Taking into account Proposition 5.5 we conclude that the spectrum of $A_{z, z^{\prime}}$ is as indicated in claim (2), and the multiplicity of $-\sigma_{m}$ equals the number of solutions in nonnegative integers of the equation

$$
2 m_{2}+3 m_{3}+\cdots=m,
$$

which proves claim (3).

Theorem 7.3. (1) The process $\boldsymbol{\omega}_{z, z^{\prime}}(t)$ constructed in Theorem 7.1 has the boundary measure $P_{z, z^{\prime}}$ as a unique stationary distribution.

(2) It is also a symmetrizing measure.

(3) The process is ergodic in the sense that for any $f \in C(\Omega)$,

$$
\lim _{t \rightarrow+\infty}\left\|T(t) f-\langle f\rangle_{P_{z, z^{\prime}}} \cdot 1\right\|=0
$$

where $\|\cdot\|$ is the norm of the Banach space $C(\Omega)$ and 1 is the constant function equal to one.

Proof. Consider the orthogonal decomposition of $\Lambda^{\circ}$ onto eigenspaces afforded by Theorem 7.2:

$$
\Lambda^{\circ}=\mathbb{R} 1 \oplus \bigoplus_{m=2}^{\infty} \Lambda_{m}^{\circ}
$$

The operator $T(t)$ leaves invariant the constant 1 and acts in $\Lambda_{m}^{\circ}$ as multiplication by $\exp \left(-\sigma_{m} t\right)$. Note that the direct sum decomposition in (7.1) is understood in purely algebraic sense: for any vector $f \in \Lambda^{\circ}$, its expansion $f=f_{0}+f_{2}+f_{3}+\ldots$ has finitely many nonzero components.

(1) The fact that $P_{z, z^{\prime}}$ is an invariant distribution follows from Proposition 1.6. To prove uniqueness we observe that if $P$ is an invariant distribution then $\langle f\rangle_{P}=0$ for any $f \in \Lambda_{m}^{\circ}, m=2,3, \ldots$ Therefore, for any $f=f_{0}+f_{2}+f_{3}+\cdots \in \Lambda^{\circ}$ with $f_{0}=c 1$ we have

$$
\langle f\rangle_{P}=\left\langle f_{0}\right\rangle_{P}=c .
$$

Since $\Lambda^{\circ}$ is dense in $C(\Omega), P$ is determined uniquely.

(2) The claim to be proved is equivalent to the fact that

$$
\int_{\Omega}\left(\bar{A}_{z, z^{\prime}} f(\omega)\right) g(\omega) P_{z, z^{\prime}}(d \omega)
$$

is symmetric under transposition $f \leftrightarrow g$, for any $f$ and $g$ in the domain of $\bar{A}_{z, z^{\prime}}$. It suffices to check this for $f$ and $g$ in $\Lambda^{\circ} \subset C(\Omega)$ and with $A_{z, z^{\prime}}$ replacing $\bar{A}_{z, z^{\prime}}$, which follows from Proposition 1.7.

(3) From the decomposition (7.1) it is evident that for any $f \in \Lambda^{\circ}$, we have $T(t) f \rightarrow f_{0}$ as $t \rightarrow+\infty$. Here the convergence holds in a finite-dimensional space invariant under the semigroup $T(t)$, hence $T(t) f$ converges to $f_{0}$ in norm, too. Together with (7.2) this proves claim (3) for $f \in \Lambda^{\circ}$. Then it is evident that it also holds for any $f \in C(\Omega)$. 
Recall that any function $f(\omega)$ from $\Lambda^{\circ} \subset C(\Omega)$ can also be viewed as a polynomial in the moment coordinates $q_{1}=q_{1}(\omega), q_{2}=q_{2}(\omega), \ldots$ With this understanding, we set, for any two functions $f, g \in \Lambda^{\circ}$

$$
\Gamma(f, g)(\omega)=\sum_{i, j=1}^{\infty} \Gamma_{i j}(\omega) \frac{\partial f}{\partial q_{i}}(\omega) \frac{\partial g}{\partial q_{j}}(\omega),
$$

where

$$
\Gamma_{i j}(\omega)=(i+1)(j+1)\left(q_{i+j}(\omega)-q_{i}(\omega) q_{j}(\omega)\right), \quad i, j=1,2, \ldots
$$

Note that the sum is actually finite, because the partial derivatives with sufficiently large indices vanish.

Theorem 7.4. For any $f, g \in \Lambda^{\circ} \subset C(\Omega)$

$$
-\int_{\Omega} A_{z, z^{\prime}} f(\omega) g(\omega) P_{z, z^{\prime}}(d \omega)=\int_{\Omega} \Gamma(f, g)(\omega) P_{z, z^{\prime}}(d \omega)
$$

The point here is that both the pre-generator $A_{z, z^{\prime}}$ and the boundary measure $P_{z, z^{\prime}}$ depend on the parameters $\left(z, z^{\prime}\right)$ while $\Gamma(f, g)$ does not: in the right-hand side, the parameters enter $P_{z, z^{\prime}}$ only.

Proof. We abbreviate $A=A_{z, z^{\prime}}$ and $\langle\cdot\rangle=\langle\cdot\rangle_{P_{z, z^{\prime}}}$. Let us show that

$$
2 \Gamma(f, g)=A(f g)-(A f) g-f(A g), \quad f, g \in \Lambda^{\circ} .
$$

Indeed, by Theorem $6.1, A$ is a second order differential operator in the moment coordinates. Therefore, its first order terms do not contribute to the right-hand side of (7.5). Writing

$$
A=\sum_{i, j=1}^{\infty} \Gamma_{i j} \frac{\partial^{2}}{\partial q_{i} \partial q_{j}}+\text { first order terms }
$$

we see that the right-hand side of (7.5) is equal to

$$
2 \sum_{i, j=1}^{\infty} \Gamma_{i j} \frac{\partial f}{\partial q_{i}} \frac{\partial g}{\partial q_{j}}
$$

which is precisely the definition of $\Gamma(f, g)$.

Next, as we already pointed out above, the expectation $\langle\cdot\rangle$ vanishes on all eigenspaces of $A$, except that corresponding to the eigenvalue 0 . Consequently $\langle\cdot\rangle$ vanishes on the range of the operator $A$. Applying the expectation to the both sides of (7.5) and using the fact that $A$ is symmetric, we get the desired formula.

Remark 7.5. Note that for any $\omega \in \Omega$, the infinite matrix $\left[\Gamma_{i j}(\omega)\right]_{i, j=1}^{\infty}$ is nonnegative definite. Indeed, recall that $q_{i}=q_{i}(\omega)$ is the $i$ th moment of the Thoma measure $\nu_{\omega}$ on $[-1,1]$. It follows that for any sequence $c_{1}, c_{2}, \ldots$ of real numbers with finitely many nonzero entries, the quadratic form $\sum_{i, j}\left(q_{i+j}-q_{i} q_{j}\right) c_{i} c_{j}$ equals the variance of the function $\sum_{i} c_{i} x^{i}$ with respect to $\nu_{\omega}$. Cf. Schmuland [S, p. 255]. It is tempting to regard the quantity $\Gamma(f, f)$ defined by $(7.3)-(7.4)$ as a square field (carré du champs). Similar expressions already appeared in works on measure-valued diffusions, see, e.g., Overbeck-Röckner-Schmuland [OvRS]. 


\section{REFERENCES}

[B] A. Borodin, Harmonic analysis on the infinite symmetric group and the Whittaker kernel, St. Petersburg Math. J. 12 (2001), no. 5, 733-759.

[BO1] A. Borodin and G. Olshanski, Point processes and the infinite symmetric group, Math. Research Lett. 5 (1998), 799-816; arXiv: math.RT/9810015.

[BO2] A. Borodin and G. Olshanski, Distributions on partitions, point processes and the hypergeometric kernel, Comm. Math. Phys. 211 (2000), 335-358; arXiv: math.RT/9904010.

[BO3] A. Borodin and G. Olshanski, Harmonic functions on multiplicative graphs and interpolation polynomials, Electronic J. Comb. 7 (2000), paper \#R28; arXiv: math/9912124.

[BO4] A. Borodin and G. Olshanski, Z-Measures on partitions, Robinson-Schensted-Knuth correspondence, and $\beta=2$ random matrix ensembles, in: Random matrix models and their applications (P. M. Bleher and A. R. Its, eds). Mathematical Sciences Research Institute Publications 40, Cambridge Univ. Press, 2001, pp. 71-94; arXiv: math/9905189.

[BO5] A. Borodin and G. Olshanski, Random partitions and the Gamma kernel, Advances in Math. 194 (2005), no. 1, 141-202; arXiv: math-ph/0305043.

[BO6] A. Borodin and G. Olshanski, Markov processes on partitions, Prob. Theory and Related Fields 135 (2006), no. 1, 84-152; arXiv: math-ph/0409075.

[BO7] A. Borodin and G. Olshanski, Meixner polynomials and random partitions, Moscow Math. J. 6 (2006), no. 4, 629-655; arXiv: math.PR/0609806.

[Ed] A. Edrei, On the generating functions of totally positive sequences II, J. Analyse Math. 2 (1952), 104-109.

[EK1] S. N. Ethier and T. G. Kurtz, The infinitely-many-neutral-alleles diffusion model, Adv. Appl. Prob. 13 (1981), 429-452.

[EK2] S. N. Ethier and T. G. Kurtz, Markov processes - Characterization and convergence, Wiley-Interscience, New York, 1986.

[FW] I. B. Frenkel and W. Wang, Virasoro algebra and wreath product convolution, J. Algebra 242 (2001), no. 2, 656-671.

[F] J. Fulman, Stein's method and Plancherel measure of the symmetric group, Trans. Amer. Math. Soc. 357 (2005), no. 2, 555-570; arXiv: math.RT/0305423.

[IO] V. Ivanov and G. Olshanski, Kerov's central limit theorem for the Plancherel measure on Young diagrams, In: Symmetric functions 2001. Surveys of developments and perspectives. Proc. NATO Advanced Study Institute (S. Fomin, editor), Kluwer, 2002, pp. 93-151; arXiv: math/0304010.

[KSK] J. G. Kemeny, J. L. Snell, and A. W. Knapp, Denumerable Markov chains, Springer, NY, 1976.

[K] S. V. Kerov, Asymptotic representation theory of the symmetric group and its applications in analysis, Amer. Math. Soc., Providence, RI, 2003, 201 pp.

[KOO] S. Kerov, A. Okounkov, G. Olshanski, The boundary of Young graph with Jack edqe multiplicities, Intern. Math. Res. Notices (1998), no. 4, 173-199; arXiv: q-alg/9703037.

[KO] S. Kerov and G. Olshanski, Polynomial functions on the set of Young diagrams, Comptes Rendus Acad. Sci. Paris Sér. I 319 (1994), 121-126.

[KOV1] S. Kerov, G. Olshanski, and A. Vershik, Harmonic analysis on the infinite symmetric group. A deformation of the regular representation, Comptes Rendus Acad. Sci. Paris, Sér. I 316 (1993), 773-778.

[KOV2] S. Kerov, G. Olshanski, and A. Vershik, Harmonic analysis on the infinite symmetric group, Invent. Math. 158 (2004), 551-642; arXiv: math.RT/0312270.

[L] J. Lamperti, Stochastic processes, A survey of the mathematical theory, Springer, 1977.

[LT] A. Lascoux and J.-Y. Thibon, Vertex operators and the class algebras of symmetric groups, J. Math. Sci. (N. Y.) 121 (2004), no. 3, 2380-2392; arXiv: math/0102041.

[Ma] I. G. Macdonald, Symmetric functions and Hall polynomials, 2nd edition, Oxford University Press, 1995.

[Ok] A. Okounkov, $S L(2)$ and $z$-measures, in: Random matrix models and their applications (P. M. Bleher and A. R. Its, eds). Mathematical Sciences Research Institute Publications 40, Cambridge Univ. Press, 2001, pp. 407-420; arXiv: math.RT/0002136.

[OO] A. Okounkov and G. Olshanski, Shifted Schur functions, Algebra i Analiz 9 (1997), no. 2, 73-146 (Russian); English translation: St. Petersburg Math. J. 9 (1998), no. 2, 239-300; arXiv: q-alg/9605042. 
[Ol1] G. Olshanski, Point processes related to the infinite symmetric group, In: The orbit method in geometry and physics: in honor of A. A. Kirillov (Ch. Duval et al.. eds.), Progress in Mathematics 213, Birkhäuser, 2003, pp. 349-393; arXiv: math.RT/9804086.

[O12] G. Olshanski, An introduction to harmonic analysis on the infinite symmetric group, In: Asymptotic combinatorics with applications to mathematical physics (A. M. Vershik, ed.), A European mathematical summer school held at the Euler Institute, St. Petersburg, Russia, July 9-20, 2001, Springer Lect. Notes Math. 1815, 2003, 127-160; arXiv: math.RT/0311369.

[OlRV] G. Olshanski, A. Regev, and A. Vershik, Frobenius-Schur functions, Studies in memory of Issai Schur (A. Joseph, A. Melnikov, R. Rentschler, eds), Progress in Mathematics 210, Birkhäuser, 2003, pp. 251-300; arXiv: math/0110077.

[OvRS] L. Overbeck, M. Röckner, and B. Schmuland, An analytic approach to Fleming-Viot processes with interactive selection, Ann. Prob. 23 (1995), 1-36.

[P] L. A. Petrov, Two-parameter family of diffusion processes in the Kingman simplex, Preprint 2007, arXiv:0708.1930 [math.PR].

[S] B. Schmuland, A result on the infinitely many neutral alleles diffusion model, J. Appl. Prob. 28 (1991), 253-267.

[T] E. Thoma, Die unzerlegbaren, positive-definiten Klassenfunktionen der abzählbar unendlichen, symmetrischen Gruppe, Math. Zeitschr. 85 (1964), 40-61.

[VK] A. M. Vershik and S. V. Kerov, Asymptotic theory of characters of the symmetric group, Funct. Anal. Appl. 15 (1981), 246-255.

[W] A. D. Wentzell, A course in the theory of stochastic processes, McGraw-Hill International, New York, 1981.

A. Borodin: Mathematics 253-37, Caltech, Pasadena, CA 91125, U.S.A.

E-mail address: borodin@caltech.edu

G. Olshanski: Dobrushin Mathematics Laboratory, Institute for Information Transmission Problems, Bolshoy Karetny 19, 127994 Moscow GSP-4, RUSSIA.

E-mail address: olsh@online.ru 